LBL --30288

DE92 000644

\title{
A Survey of Air Flow Models for Multizone Structures
}

\author{
Helmut E. Feustel and Juergen Dieris
}

Indoor Environment Program

Applied Science Division

Lawrence Berkeley Laboratory

Berkeley, CA 94720, USA

March 1991

This report has been reproduced directly from the best available copy

This work was jointly supported by Carl-Duisberg-Gesellschaft e.V., Koeln, FRG, and by the Assistant Secretary for Conservation and Renewable Energy, Office of Building and Community Systems, Building Systems Division of the U.S. Department of Energy under Contract No. DE-AC03-76SF00098.

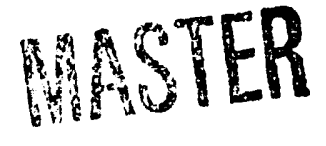


LBL - 30288

\title{
A Survey of Air Flow Models for Multizone Structures
}

\begin{abstract}
Air flow models are used to simulate the rates of incoming and outgoing air flows for a building with known leakage under given weather and shielding conditions. Additional information about the flow paths and air-mass flows inside the building can only be made by using multizone air flow models. In order to obtain more information on multizone air flow models, a literature review was performed in 1984 [1]. A second literature review and a questionnaire survey performed in 1989, revealed the existence of 50 multizone air flow models, all developed since 1966, two of which are still under development. All these programs use similar flow equations for crack flow, but differ in the versatility to describe the full range of flow phenomena and the algorithm provided for solving the set of nonlinear equations. This literature review has found that newer models are able to describe and simulate the ventilation systems and interrelation of mechanical and natural ventilation.
\end{abstract}




\section{Introduction}

Air flow models aro used to simulate the rates of incoming and outgoing air flows for a building with known leakage under given weather and shielding conditions. Air How models can be divided into two main categories, single zone models and multi-zone models. Single zone models assume that the structure can be described by a single, well mixed zone. The major application for this model type is the single-story, single-family house with no internal partitions (e.g., all internal doors are open). A large number of buildings, however, have structures that would characterize them more accurately as multizone structures, more detailed models have been developed, which also take internal partitions into account. Figure 1 shows a simple multizone network [2].

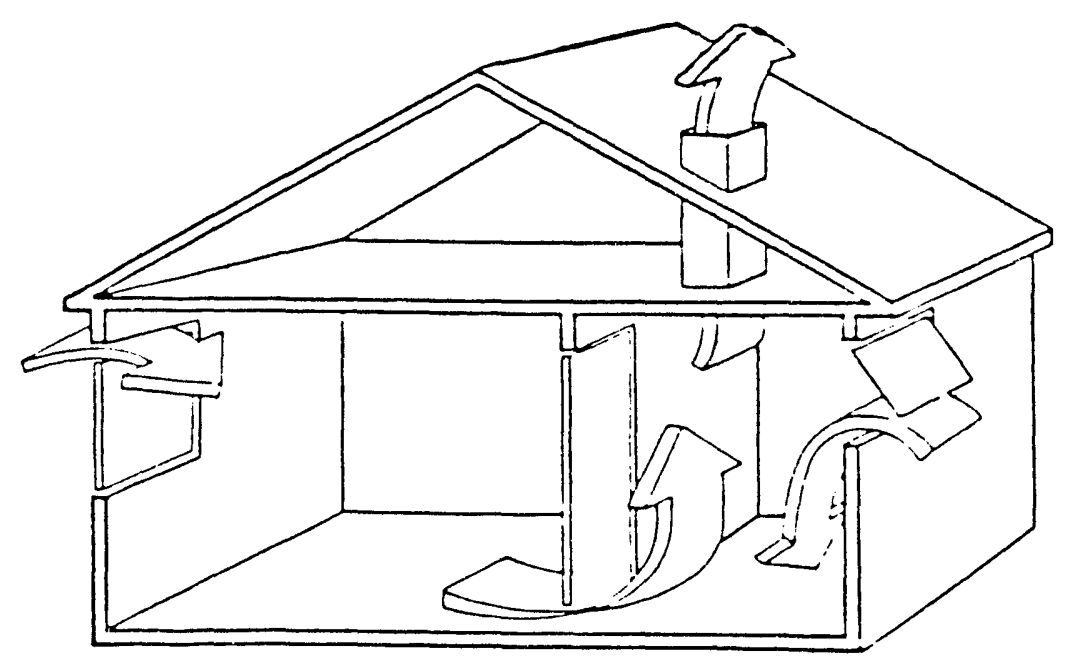

Figure 1: Simple Multizone Network

Multizone air flow network models deal with the complexity of flows in a building by recognizing the effects of internal flow restrictions. They require extensiv information about flow characteristics and pressure distributions and. in many cases, are too complex to justify their use in predicting flow for simple structures such as single-family residences [3].

As for their single-zone counterparts. these models are based on the mass-balance equation. Unlike the single-zone apiroach, where there is only one internal pressure to be determined, the multizone models must determine one pressure for each of the zones. This adds considerably to the complexity of the mumerical solvine alsorithm. but by the same token. the multizone approach offers great potential in analyzing infiltration and ventilation air llow distribution. 
The advantage of multizone models, besides being able to simulate infiltration in larger buildings, is their ability to calculate mass flow interactions between the different zones. Understanding the air-mass flow in buildings is important for several reasons :

- exchange of outside air with inside air is necessary for building ventilation

- energy consumed to heat or cool the incoming air to inside comfort temperature

- air needed for combustion

- airborne particles and germs transported by air flow in buildings

- smoke distribution in case of fire.

The air-mass flow distribution for a given building is caused by pressure differences, whether evoked by wind, thermal buoyancy, mechanical ventilation systems or a combination of those. The distribution of openings in the building shell and the inner paths also influence the air flow. The openings can be varied by the inhabitants. This can lead to significant differences in the pressure distribution inside the building. Figure 2 shows in detail the influence of pressure distribution on the air-mass flow distribution.

In terms of air mass flow, buildings are complicated interlacing systems of flow paths. In this grid-system the joints are the rooms of the building and the connections between the joints simulate the flow paths including the flow resistances caused by open or closed doors and windows or background leakage of the partitions. The boundary conditions for the pressure can be described by the grid points outside the building. The wind pressure distribution depends on the velocity and the direction of the wind, the terrain surrounding of the building, and the shape of the building. If the physical interrelationship between the flow resistance and the air flow is known for all flow paths, the air flow distribution for tile building can be calculated, as long as there is no density difference between outside and inside air. Differences in density of the air, due to differences between outside and inside air temperatures or moisture content, cause further pressures in the vertical direction, again influencing the air mass flow.

Mechanical ventilation can also be included in this network. The duct system can be treated like the other flow paths in the building. The advantage for calculating air flow distribution effects of mechanical ventilation systems is that the duct path ways, as well as their connections with the building, are known. In the case of mechanical ventilation systems the fan can be described as the source of pressure differences; lifting the pressure level between two joints according to the characteristic curve of the fan.

Because of the non-linear dependency of the volume flow rate on the pressure difference, the pressure distribution for a building can be calculated only by using a method of iterations. Large quantities of computer storage was necessary for the early models to describe buildings with arbitrary floor plans and to solve the set of non-linear equations. 


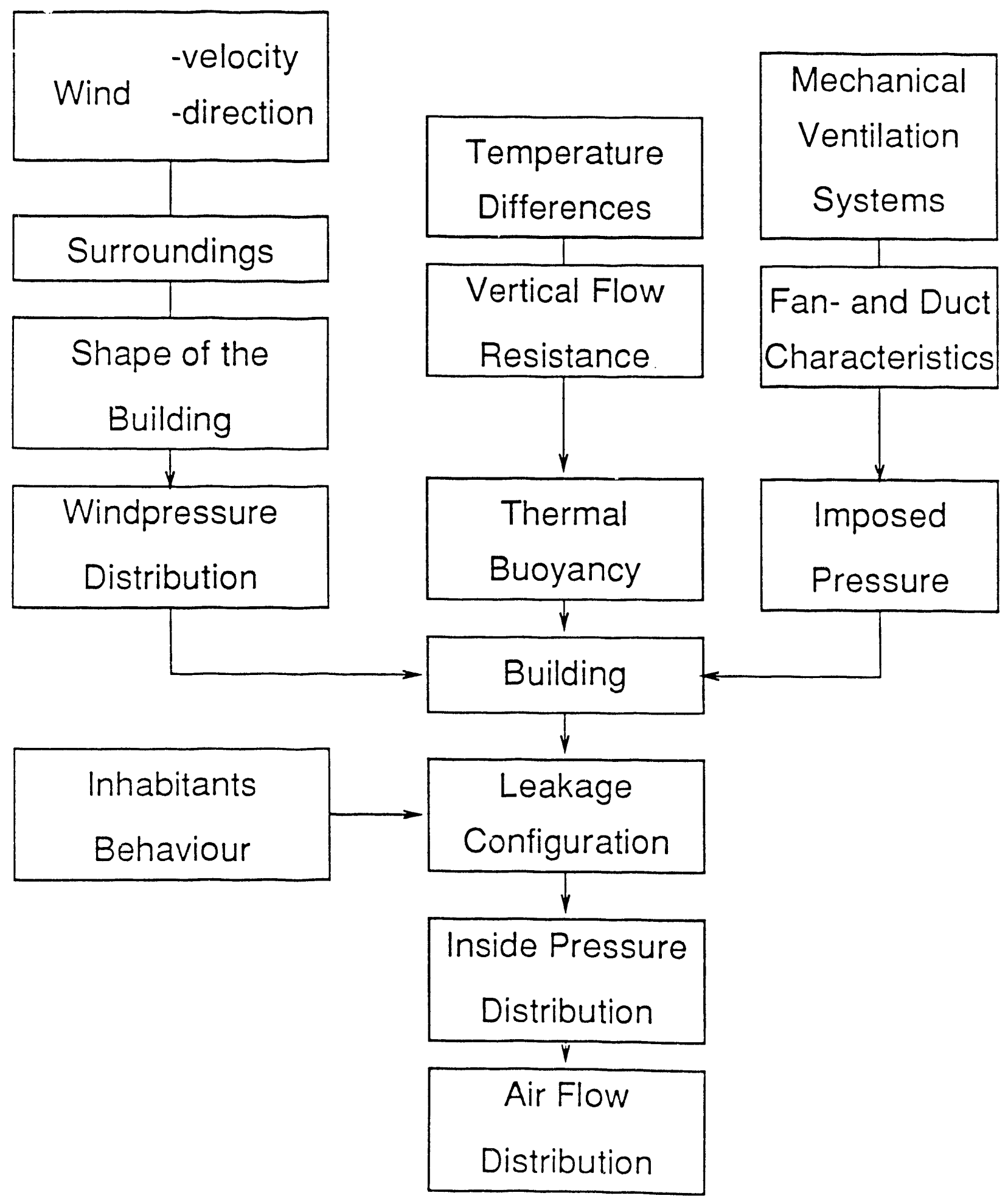

Figure 2: Influence of Pressure Distribution on the Air-Mass Flow in Buildings 


\section{Literature Review and Questionnaire Survey}

A literature review undertaken in 1984 [1] revealed 26 papers describing 15 different multizone infiltration models which had been developed in eight countries. A recent questionnaire survey produced additional information about the status of network models. One of the first models we found was Jackman's model "LEAK" [4] which was published in 1970. In 1974 it was followed by the NRCC modei [5], which was the first one available to interested parties. Indeed, this numerical tool is probably still the most widely used multizone infiltration model.

Several multizone models were developed in the aftermath of the oil price crises. Between 1975 and 1977 a series of steps were taken at Technische Universitaet Berlin to develop such a model. This resulted in the program STROM [6] which was later extended to handle HVAC-systems and has recently been rewritten to improve the solver and to be combined with a thermal building simulation model [7]. Concurrent with STROM, ELA 4 [8] was developed. The models VENT 1 and VENT 2 [9] as well as BREEZE [10] were developed in the late 1970's by researchers from British Gas and the Building Research Establishment. In the early 1980's, the first versions of Nantka's INFILTRATION \& VENTILATION [11], Walton's AIRNET [12] and Herrlin's MOVECOMP [13] appeared. The latter two programs were the first to address the mathematical problem of solving the set of non-linear equations for zones with very different leakage characteristics. Open doorways in otherwise tight constructions are the main cause of significant problems in convergence. This has been solved by introducing underrelaxation factors to the well known Newton method.

KGVCP from Hayakawa dates back to 1979 [14]. More recent work done in Japan has been published by Ishida and Udagowo [15] as well as Hayashi and Urano [16], Sasaki [17], Okuyama [18] and Matsumoto and Yoshino. [19].

France has also developed several multizone models; the recent survey unveiled models from CSTB, INSA [20] and EDF. From Brazil, Melo's model FLOW2 [21] has been discovered.

The latest development in infiltration modeling is the COMIS model [22]. In a twelve month period ten scientists from nine countries, working together at Lawrence Berkeley Laboratory, developed a multizone model on a modular base. Becalise of its modular structure COMIS is designed to expand its capability to simulate buildings. To accomplish a "user-friendly" program, special emphasis was given to input and output routines. Support of the international group by IEA's Air Infiltration and Ventilation Centre will help the wide distribution of this model to all interested parties. COMIS can be used as a stand-alone infiltration model with input and output features, or as an infiltration module for thermal building sinulation programs. It also serves as a module library.

One of the major tasks for the program user is to find a method of determining the wind pressure distribution for a building. This can be done by measuring the wind pressure distribution on a scale model in a wind tunnel or by using measured data from available literature. Only few attempts have been made to 
calculate the wind pressure distribution. In order to calculate the Cp-distribution for buildings, the COMIS group worked on a method based on a parametrical study, to determine the Cp-values [23].

Crack flow, large openings and mechanical ventilation systems can be simulated by some of the models. Furthermore, additional flows, i.e., simultaneous two way flow at large openings and wind turbulence effect at single-sided windows etc., were studied.

Airflow rates through doorways, windows and other common large openings are significant ways in which air, pollutants and thermal energy are transferred from one "zone of a building to another.

In the previous survey of multizone infiltration models, however, none of the described codes were able to solve this problem in any other way than to divide the large opening into a series of small ones described by crack flow equations.

Therefore, COMIS's contribution to this fundamental problem was to describe the physical problem, review the various solutions developed in the literature and compare these solutions using both a numerical and a physical point of view.

In most cases the temperature of the air in a crack is quite different from the temperatures of the zones on either side of the crack. Furthermore, air leakage performance measurements are usually performed in a certain temperature condition, but results are used at different temperatures. The temperature variation, however, has a big influence on the air leakage flow due to changes in the air viscosity and air density. Unfortunately, almost all the models dealing with air leakage characteristics ignore this phenomenon.

HVAC-Systems (Heating, Ventilating and Air-Conditioning Systems) are composed of ducts, duct fittings, junctions, fans, air filters, heating and cooling coils, air-to-air heat exchangers, flow controllers, etc. Several modeling programs for ventilating systems were developed.

Calculating the infiltration and ventilation flow rates requires the solution of a non-linear system of equations. The main task is to find an efficient solving method. The starting point is the Newton-Raphson method, with derivatives, operating on a node-oriented network which, in most cases, quickly brings about the convergence of the system of equations.

Although network computer models are available, there is an obvious need for simplified multichamber infiltration models capable of providing the same accuracy as the established single-cell models. The extended crack model is the simplest multizone model. It is used to design conditioning loads and size the conditioning equipment [2.1]. The method is based on the single-zone crack model and has been refined to multizone applications by also taking into consideration the crack flow through internal partitions. Buildings are characterized according to their cross flow and stack flow capabilities. The stack pressures of the core zones for multistory structures are pre-calculated for design weather conditions and published in look-up tables. Together with pressures due to the effect of wind 
and permeability distribution this enables a user to calculate the infiltration for each of the outside zones.

The simplified multizone infiltration model developed at LBL [25] is able to calculate the air flow distribution for arbitrary structures in any given weather condition. The basic idea is to determine the flow network and to calculate the resultant air permeability of a zone from the combination of flow paths arranged in series and/or parallel. The use of these equations assumes that all permeabilities have the same flow characteristics and, therefore, the same exponent, $n$.

Air flows caused by separate mechanisms (such as wind and thermal buoyancy) are not able to be added because the flow rates are not linearly proportional to the pressure differences. To superimpose the flows, it is necessary to add the pressures.

Several lumped parameters reflecting the different permeability distributions of the building's envelope and the flow resistances inside the building were introduced to describe the air flow distribution. To keep the model simple no massbalance equation is used to predict the air flows. Because of the pre-calculation of the simplified network, the model gives a comprehensive understanding of the flow characteristics of the structure under investigation. The model is simple to use and requires only simple mathematical tools, e.g., pocket calculator.

In terms of the total number of returned questionnaires this survey represents the most comprehensive review of current research in developed multizone infiltration models. The questionnaires were mailed to addresses provided by the Air Infiltration and Ventilation Centre $[26,27]$. Fifty multizone infiltration models developed in 16 countries are represented in this survey. In addition to the general references more than 60 paper describing the programs in detail are listed in Appendix B together with the names and addresses of the authors. Of those models for which questionnaires were not returned, information was collected from the reviewed literature.

In cases where the reviewed paper did not give a reasonable answer to a certain problem or where information was missing from a returned questionnaire, a question mark was entered in the list of models.

The summarized list of multizone air flow models found in the literature are reproduced in Appendix A. The current addresses of the authors including their references are shown in Appendix B. Equations are reproduced separately in Appendix C. 


\section{Discussion}

The survey of multizone network models is summarized in Table 1. It shows that 34 models have been written in FORTRAN (FORTRAN IV (F IV), FORTRAN V ( $F \mathrm{~V}$ ), and FORTRAN 77 ( $F$ 77). The programming language BASIC has been used four times; followed by HPL with three and " $\mathrm{C}$ " with two applications. PASCAL and dBaseIV have been used for the development of one model each.

Among the analyzed models it appeared that 14 were developed on main frame computers, 15 on PC's and 11 on workstations.

In the past large amounts of computer storage was necessary, when calculating the air flow distribution of more complicated buildings. Nowadays most programs use solvers which reduce the space requirement, e.g., band matrices or the skyline method. The Newton method is the most common tool used to solve the set of nonlinear equations. The majority of the programs use a technique based on Newton-Raphson. Other Algorithms are used less frequently.

The limits of zones and openings per zone a model can handle depends on computer storage. Due to the limited availability of computer storage most of the earlier models were not able to handle more than 100 zones. Models developed later are able to handle an unlimited number of zones.

Approximately $1 / 3$ of the models do not have an interactive input option and the output features listed show that only few models use graphical output or statistical functions rather than files comparable to the arrays used by the model. These features are an indication of a user unfriendly modiol. CAD - Input and 3 - D building description are not necessary to run the program, but would increase the user-friendliness. Unfortunately, these input features belong to the "special design", and are rarely integrated in these kind of models.

Only 19 models take into consideration occupant schedules which can lead to significant differences in the pressure distribution.

The output features show that the graphical output and the statistical functions are less applied than files used by the model.

Twentyfour of the models use a separate input program and twenty models are based on a modular structure. A modular structure might be more advantageous for further development, because the model would be easier to expand. A combination with other models would also be easier to implement.

Fifteen of the models allow a combination with a thermal model and thirteen models are coupled with a pollution model.

Less than one third (15) of the modcis are available to third parties. Some models are not available before completing and testing. Fifteen models are not available to the public and are written as research tools, rather than for the use of professional engineers or architects. 


\begin{tabular}{|c|c|c|c|}
\hline $\begin{array}{l}\text { Program Language : } \\
\text { FORTRAN } \\
\text { BASIC } \\
\text { PASCAL } \\
\text { C } \\
\text { HPL } \\
\text { dBaselV } \\
\end{array}$ & $\begin{array}{c}34 \\
4 \\
1 \\
2 \\
3 \\
1\end{array}$ & & \\
\hline $\begin{array}{l}\text { Computer Type : } \\
\text { Main Frame Computer } \\
\text { Work Station } \\
\text { Personal Computer } \\
\end{array}$ & $\begin{array}{l}14 \\
11 \\
15 \\
\end{array}$ & & \\
\hline $\begin{array}{l}\text { Solver : } \\
\text { Hardy - Cross - Method } \\
\text { Newton - Raphson Method } \\
\text { Levenberg - Marquard - Method } \\
\text { Brown - Conte - Method } \\
\text { Secant - Method } \\
\text { Rule "falsi" } \\
\text { Gaussian - Elimination } \\
\text { Beta - Method by Newmark } \\
\end{array}$ & $\begin{array}{c}1 \\
25 \\
1 \\
1 \\
3 \\
1 \\
1 \\
1\end{array}$ & & \\
\hline $\begin{array}{l}\text { Limits : } \\
\text { max. number of zones } \\
\text { max. number of opening/zone } \\
\text { max. number of shafts/corridors/floors } \\
\text { max. number of mechanical ventilation system }\end{array}$ & $\begin{array}{c}\leq 100 \\
18 \\
17 \\
17 \\
18 \\
\end{array}$ & $\begin{array}{l}>100 \\
7 \\
3 \\
2 \\
2 \\
\end{array}$ & $\begin{array}{c}\text { unlimited } \\
18 \\
23 \\
23 \\
20 \\
\end{array}$ \\
\hline $\begin{array}{l}\text { Input Features : } \\
\text { interactive input } \\
\text { CAD input } \\
\text { weather data from weather files } \\
3 \text { - D building description } \\
\text { schedules (e.g., occupants) } \\
\end{array}$ & $\begin{array}{c}\text { yes } \\
15 \\
1 \\
24 \\
10 \\
19 \\
\end{array}$ & $\begin{array}{l}\text { no } \\
21 \\
30 \\
14 \\
26 \\
17\end{array}$ & \begin{tabular}{|c|} 
not specified \\
14 \\
19 \\
13 \\
14 \\
14 \\
\end{tabular} \\
\hline $\begin{array}{l}\text { Output Features: } \\
\text { file of arrays used by the model } \\
\text { graphical output } \\
\text { statistical functions }\end{array}$ & $\begin{array}{c}\text { yes } \\
29 \\
12 \\
5 \\
\end{array}$ & $\begin{array}{c}\text { no } \\
6 \\
23 \\
29 \\
\end{array}$ & \begin{tabular}{|c|} 
not specified \\
15 \\
15 \\
16 \\
\end{tabular} \\
\hline $\begin{array}{l}\text { Structure : } \\
\text { separate input program } \\
\text { modular structure }\end{array}$ & $\begin{array}{c}\text { yes } \\
24 \\
20 \\
\end{array}$ & $\begin{array}{l}\text { no } \\
14 \\
14 \\
\end{array}$ & \begin{tabular}{|c|} 
not specified \\
12 \\
16 \\
\end{tabular} \\
\hline $\begin{array}{l}\text { Combination with other models : } \\
\text { combined with thermal model } \\
\text { combined with pollution model }\end{array}$ & $\begin{array}{c}\text { yes } \\
15 \\
13\end{array}$ & $\begin{array}{l}\text { no } \\
24 \\
26\end{array}$ & $\begin{array}{c}\text { not specified } \\
11 \\
11 \\
\end{array}$ \\
\hline $\begin{array}{l}\text { Availability : } \\
\text { program available to third parties }\end{array}$ & $\begin{array}{r}\text { no } \\
15\end{array}$ & $\begin{array}{c}\text { yes } \\
15\end{array}$ & $\begin{array}{c}\text { yes, but } \\
12\end{array}$ \\
\hline
\end{tabular}




\section{Conclusion}

This literature review and the questionnaire survey show that an extensive research effort has been made in the last five years to develop an air flow model for multizone structures. Especially the maximum number of zones the models can handle has been increased due to reduced computer storage requirements. Some of the models have been extended with special input and output features.

Fifteen of the models are available to third parties and fifteen models have been written as research tools, and are difficult to use. This might be the reason they are not available to third parties.

The development of multizone infiltration and ventilation models shows a relatively slow evolution. Lack of exchange of information, restricted distribution of models, poor documentation, and lack of a flexible structure are probably the reasons why models developed in the early seventies are not very different from those developed in the late eighties.

Although several of the models discovered during this survey serve a particular purpose, they could have been developed using existing models. The COMIS workshop was trying to overcome these problems by creating a multizone infiltration model with a modular structure which will allow modules to be changed easily. The availability of the program and its documentation together with the international authorship should help to establish COMIS as an infiltration standard on which specific applications can be built.

\section{Future Outlook}

Along with stand-alone infiltration models, network models will also find their way into thermal building simulation models. With the expected advances in the development of the next generation of building simulation programs, infiltration modules will be needed for implementation in the program libraries.

Future tasks include the development of methods to determine the required input parameters, especially the wind pressure distribution. Further work must be done through sensitivity studies to reduce the input requirement and to increase userfriendliness by using the output features of the PC's.

Validation of the models is another essential task. In order to understand physical phenomena related to transport mechanism in buildings and to develop numerical descriptions, measurements must first be performed under steady state conditions. It is necessary, in order to measure mass flow transport mechanism accurately, to be able to control the pressure level and its fluctuation for each of the outside walls. This is only possible if the test building itself is located in a building. Such a test facility would not only validate air flow models as a whole, but would also help to validate the tracer gas techniques used to validate infiltration models in field experiments. 
Recent developments in measurement techniques open new possibilities to study physical phenomena and use the results for comparison with results from the model. Some data sets for evaluation purposes have been produced based on insitu measurements of heavy instrumented buildings. Before these data sets can be used for model evaluation, however, internal model comparisons based on benchmark buildings have to be performed.

The Energy Conservation in Buildings and Community Systems Program of the International Energy Agency adopted a new working group (Annex 23) to study physical phenomena causing air flow and pollutant transport (e.g., moisture) in multizone buildings and to develop modules to be integrated in a multizone air flow modeling system. The system itself shall be user-friendly and structured to be incorporable in thermal building simulation models. Furthermore, special emphasis shall be given to provide data necessary to use the system (e.g., wind pressure distribution, default values for leakage of building components, material properties like absorbtion and desorption). The comparison between results from the model and from in-situ tests is an important part of this annex.

Close cooperation is envisaged, with regard to state-of-the art reviews, data collection, coordination of work, e.g., defining cases for evaluation purposes with other pertinent projects. The work to be carried out will be complementary with the above mentioned Annexes.

The Air Infiltration and Ventilation Centre will, as part of its on-going work plan, act as a vehicle for disseminating the results of this particular Annex. A data base for evaluation purposes is going to be prepared by AIVC. The Centre has already started to collect wind pressure data and leakage data.

\section{Acknowledgements}

We would like to acknowledge the program authors for their cooperation in responding to our questionnaires, for without this information the different programs could not have been summarized.

This work was jointly supported by the Carl-Duisberg-Gesellschaft, Koeln, Federal Republic of Germany, and the Assistant Secretary for Conservation and Renewable Energy, Office of Building and Community Systems, Building Systems Division of the U.S. Department of Energy under Contract No. DE-AC0376SF00098. 


\section{Referunces}

1. Feustel, H.E. and V.M. Kendon: "Infiltration Models for Multicellular Structures - a Literature Review", Energy and Buildings, Vol 8, No. 2, 1985, Lawrence Berkeley Laboratory, LBL-Feport No. 17588, April 1985

2. Liddament, M.: "Air Infiltration Calculation Techniques - An Applications Guide", Air Infiltration and Ventilation Centre, June 1986

3. ASHRAE 1985 Fiandbook of Fundamentals, Chapter 22, American Society of Heating, Refrigerating and Air-Conditioning Engineers, Atlanta, GA, 1985

4. Jackman, P.J.: "A Study of Natural Ventilation of Tall Office Buildings", Journal of Institution of Heating and Ventilating Engineers, Vol 38, 1970

5. Sander, D.M. "FORTRAN IV Program to calculate Air Infiltration in Buildings", DBR Computer Program No. 37, National Research Council Canada, 1984

6. Feustel, H.E.: "Entwicklung eines FORTRAN Programms zur Bestimmung der Luftstromverteilung in Gebaeuden", Thesis, Technische Universitaet Berlin, 1977

7. Jianxiong Guo: "Weiterentwicklung des Programms mit dem Ergebnis einer Beruecksichtigung thermischer Druckdifferenzen auch innerhalb eines Geschosses and einer spuerbaren Verringerung der Rechenzeiten", Interner Institutsbericht, Technische Universitaet Berlin, 1088

8. de Gids, W.F.: "Calculaticn Method for the Natural Ventilation of Buildings", TNO Research Institute for Environmental Hygiene, Delft, 1977

9. Etheridge, D.IV. and D.K. Alexander: "The British Gas Multi-Cell Model for Calculating Ventilation", ASHRAE Transactions, Vol. 86, Part II, 1980

10. Evers, E. and A. Waterhouse: "A Computer Model for Analysing Smoke Movement in Buildings", Building Research Establishment, 1978

11. Nantka, M.: "A Numerical Method for Air Change Rate of Buildings Calculated" in Proceedings, 5th International Conference on HVAC, Praha, 1981

12. Walton, G.N.: "Thermal Analysis Research Program Reference Manual", NBSIR 83-2655, U.S. Department of Commerce, 1983 
13. Herrlin, M.K.: "MOVECOMP: A static Multicell Air Flow Model", ASHRAE Transactions, Vol 91, Part 2B, 1085

14. Hayakawa, S and S. Togari: "Flow Rate Variation of Air Conditioning Systems due to Stack Effect and opening a Window", Kajima Institute of Construction Technology, Japan, Report Nr. 30, 1979

15. Ishida, K. and M. Udagowo: "Validation of the Ventilation Net Work Model for the Estimation of Room Temperatures and Heat Load of Residential Buildings with Measured Data", Transactions of the Architectural Institute of Japan, 1988

16. Hayashi, T., Y. Urano, T. Watanabe and Y. Ryu: "Passive System Simulation Program PSSP and its Applications", in Proceedings, "Building Energy Simulation Conference 1985, Seattle, 1985

17. Sasaki, T.: "Der Lueftungszustand von der Luftschicht durch WindUaruhe", Transactions of the Architectural Institute of Japan, No. 372, 1987

18. Okuyama, H.: "Network Numerical Analysis Method for Heat Transfer and Air Flow in Buildings", in Proceedings, 17th Symposium by the Committee of Building Heat Transfer, Building Environment Committee of Architectural Institute of Janan, 1987

19. Matsumoto, M. and H. Yoshino: "A Calculation Method for Predicting Air Pollution in Multi-Celled Buildings", The Meeting of the Tohoku Branch of the Architectural Institute of Japan, 1988

20. Cacavelli, D., J.J. Roux; and F. Allard: "A simplified Approach of Air Infiltration in Multizone Buildings", in Proceedings, "9th AIVC Conference", Gent, 1988

21. Melo, C.: "FLOW - An Algorithm for Calculating Air Infiltration into Buildings", in Proceedings, ICBEM'87 International Congress on Building Energy Management, Lausanne, 1987

22. Feustel, H.E., F. Allard, V.B. Dorer, M. Grosso, M. Herrlin, M. Liu, J.C. Phaff, Y. Utsumi, and H. Yoshino: "The COMIS Infiltration Model", in Proceedings, "Building Simulation '89, The International Building Performance Simulation Association", Vancouver, 1089.

23. Feustel, H.E. and A. Raynor-Hoosen (Editors): "Fundamentals of the Multizone Air Flow Model - COMIS", Air Infiltration and Ventilation Centre, 
Warwick, Technical Note 29, May 1990

24. German Standard DIN 4701: "Regeln fuer die Berechnung des Waermebedarfs von Gebaeuden", Teil 1: Grundlagen der Berechnung Teil 2: Tabellen, Bilder, Algorithmen Beuth Verlag Berlin, Berlin, 1983

25. Feustel, H.E. and M.H. Sherman: "A Simplified Model for Predicting Air Flow in Multizone Structures", Energy and Buildings, Vol 13, No. 3, 1989

26. Charlesworth, P.: "1986 Survey of Current Research into Air Infiltration and Related Air Quality Problems in Buildings", Air Infiltration and Ventilation Centre, Technical Note 19, December 1986

27. Blacknell, J.: "A Subject Analysis of the ATVC's Bibliographic Database AIRBASE (6th edition)", Air Infiltration and Ventilation Centre, Technical Note 25, January 1989 


\begin{abstract}
APPENDICES
The following appendices describe the reviewed literature and the results of the questionnaire survey in a tabular form. Fifty different multizone air flow models are listed in Appendix A. A question mark was entered in the table of models where the reviewed paper did not give a reasonable answer to a certain problem or where information was missing from the returned questionnaire. The authors of the models can be fourd in Appendix B. Together with the authors of the computer models and their current addresses, more than 60 papers describing the models in detail are listed in Appendix B. Equations used by the models as well as a nomenclature are shown separately in Appendix C.
\end{abstract}




\begin{tabular}{|c|c|c|c|c|c|c|c|c|c|}
\hline$=$ & 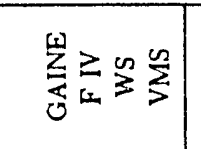 & ¿ & 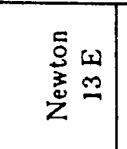 & 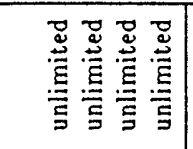 & 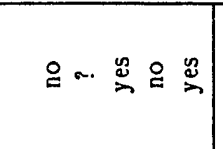 & 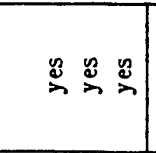 & 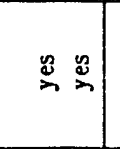 & 음 & $\stackrel{\circ}{\circ}$ \\
\hline 인 & 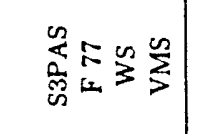 & 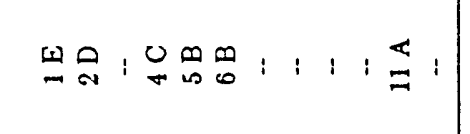 & 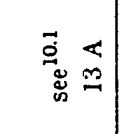 & 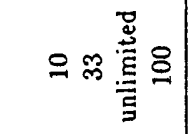 & 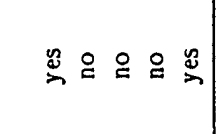 & 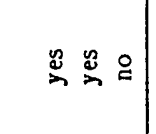 & $\stackrel{8}{2}$ & $\stackrel{\leftrightarrow}{2}$ & - \\
\hline$\infty$ & $\stackrel{\omega}{\frac{\omega}{2}}=\Sigma:$ & 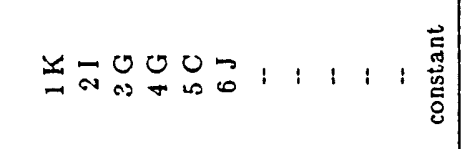 & $\sum_{i}^{n} \sum_{i}^{\infty}$ & 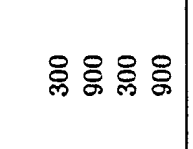 & 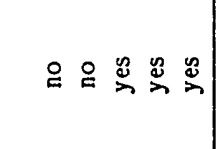 & 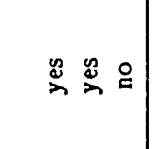 & 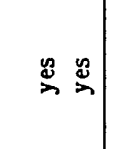 & $\stackrel{\Xi}{\varpi}$ & 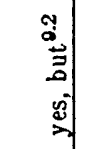 \\
\hline$\infty$ & 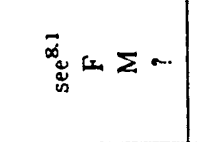 & 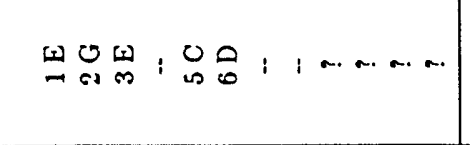 & 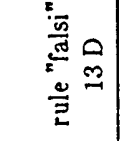 & 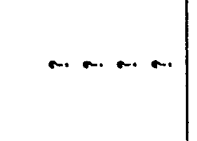 & 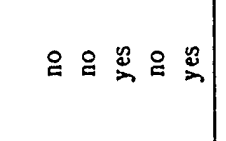 & 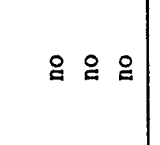 & $\stackrel{8}{\square}$ & $\cong$ & 인 \\
\hline r & 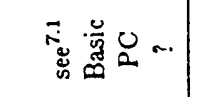 & 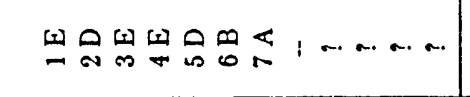 & $\stackrel{\infty}{\sim}$ & 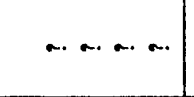 & 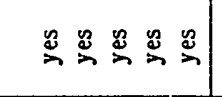 & 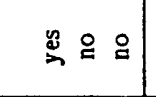 & 吕 & 욤 & 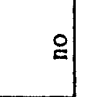 \\
\hline 0 & 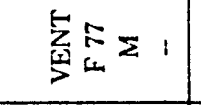 & 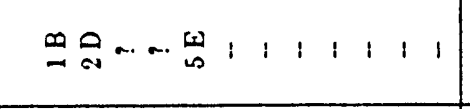 & $\cdots \stackrel{\mathbb{m}}{\dddot{m}}$ & $\infty \cong x$ & 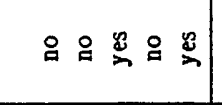 & 욤이 & $\stackrel{8}{2}$ & $\therefore \&$ & 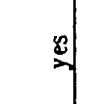 \\
\hline is & 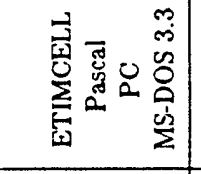 & 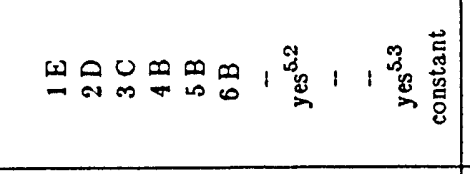 & 范 $\cdots$ & 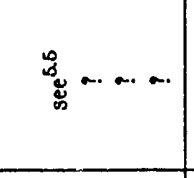 & 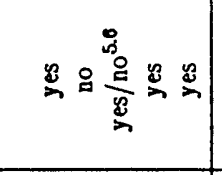 & $\stackrel{9}{2} \div$ & $\mathscr{2}$ & ஃ & 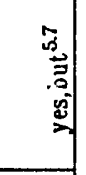 \\
\hline+ & 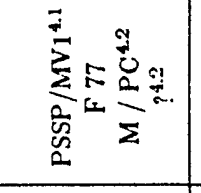 & 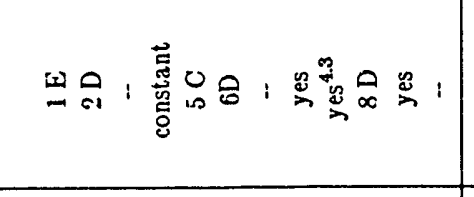 & 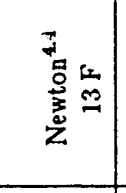 & 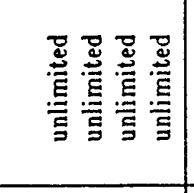 & 욤요 & 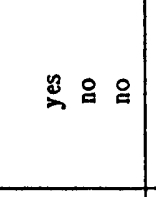 & $\stackrel{8}{2}$ & 80 & $y_{0}^{2}$ \\
\hline$\infty$ & 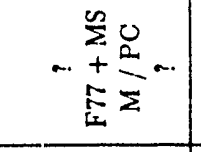 & 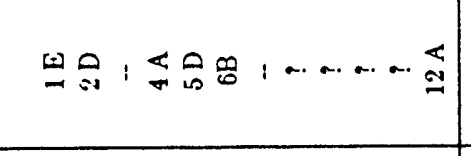 & $\begin{array}{ll}2 & 0 \\
z & 0\end{array}$ & 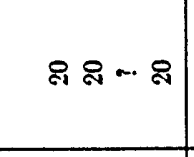 & 욤 & $\stackrel{2}{2}$ & 8 & $\stackrel{8}{2}$ & $\stackrel{\circ}{\circ}$ \\
\hline o. & ब్ & $\varrho \triangleq \triangleq ;$ ส & 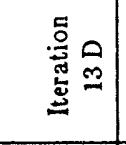 & 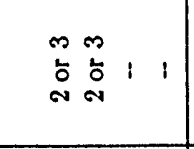 & 응응 & 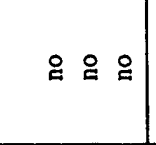 & $\cong \cong$ & $\therefore$ & 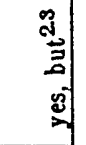 \\
\hline-1 & 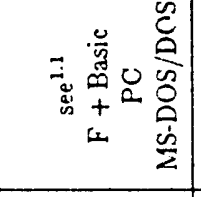 & 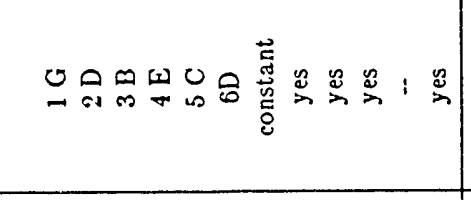 & $\begin{array}{ll}2 & 0 \\
z & 9\end{array}$ & 요요 & 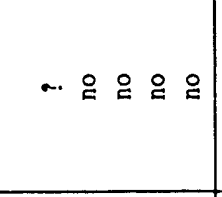 & 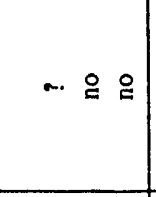 & $\cdots$ & 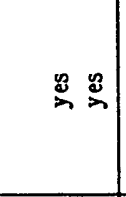 & 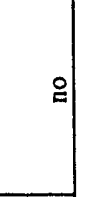 \\
\hline 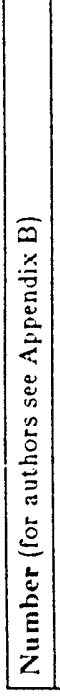 & 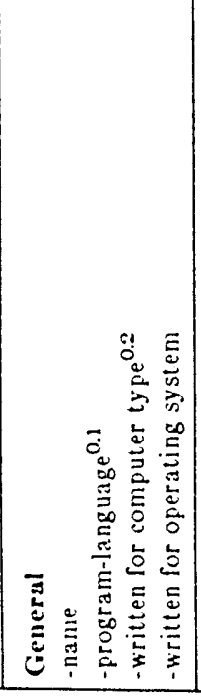 & 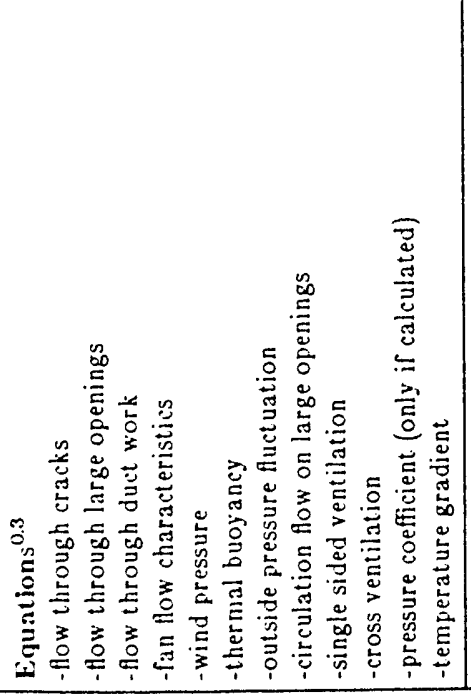 & 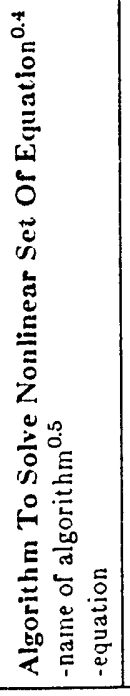 & 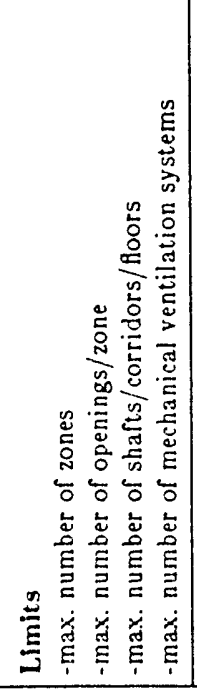 & 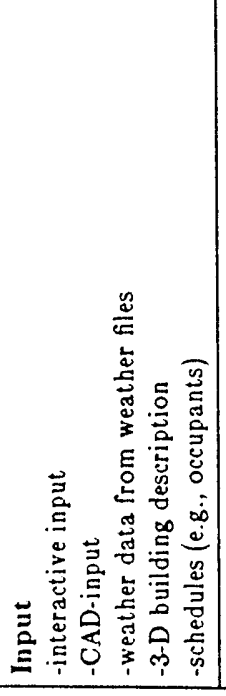 & 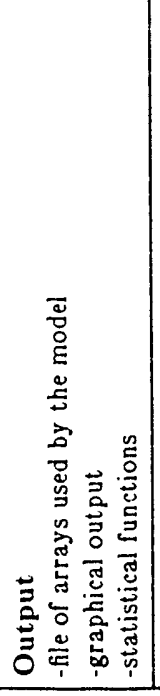 & 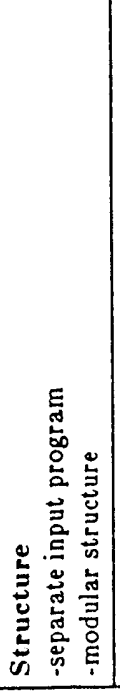 & 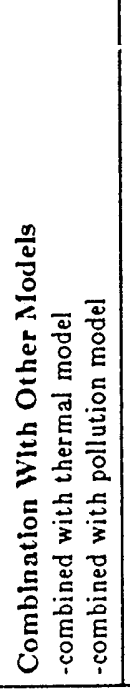 & 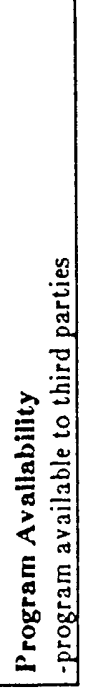 \\
\hline
\end{tabular}




\begin{tabular}{|c|c|c|c|c|c|c|c|c|c|}
\hline สี & 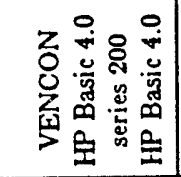 & 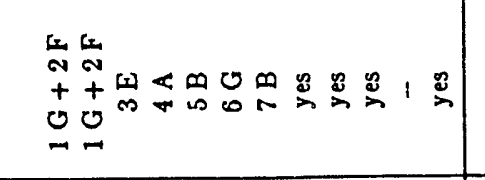 & 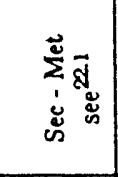 & 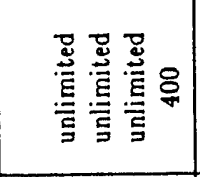 & 용ㅇㅇ & 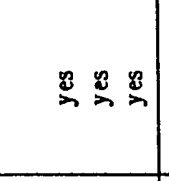 & $\stackrel{\square}{\square}$ & 80 & ㅇ \\
\hline$\vec{a}$ & $: 4 \frac{0}{0} \frac{0}{0}$ & 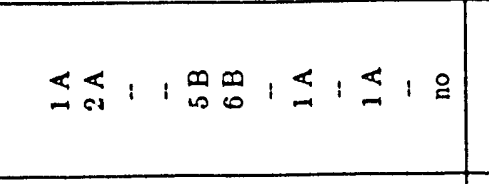 & $\begin{array}{ll}x & 1 \\
z & 1\end{array}$ & 导导导 & $\stackrel{丶}{\check{2}}$ 욤 & 요 & $\therefore:$ & $\stackrel{2}{\circ}$ & \& \\
\hline ฉ & 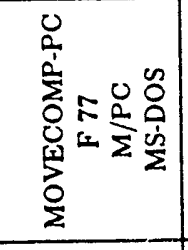 & 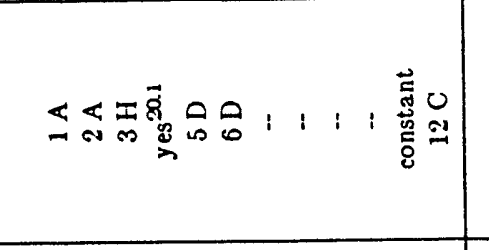 & 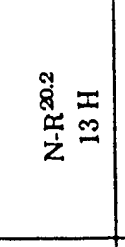 & 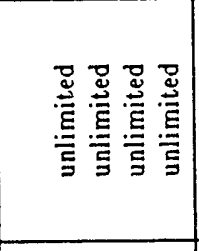 & 웜ㅇㅇㅣ & $\stackrel{\Xi}{\check{D}}$ & $\stackrel{\mathscr{D}}{\stackrel{\varpi}{二}}$ & 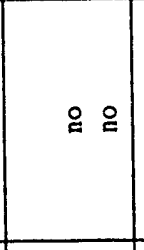 & 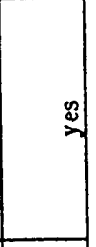 \\
\hline 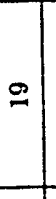 & 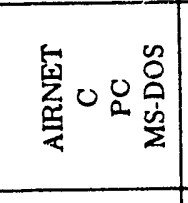 & 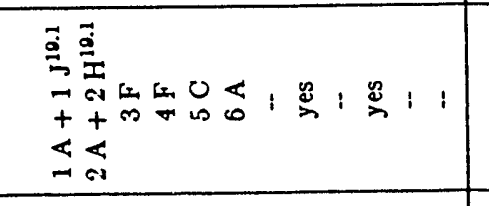 & 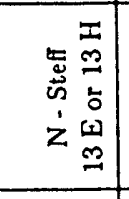 & 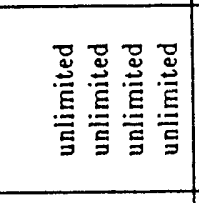 & 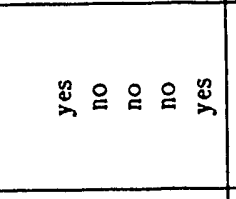 & $\stackrel{\circ}{\circ} \circ$ & $\stackrel{\mathscr{d}}{\infty}$ & 음 & $\stackrel{\$}{2}$ \\
\hline$\infty$ & 芳下 & 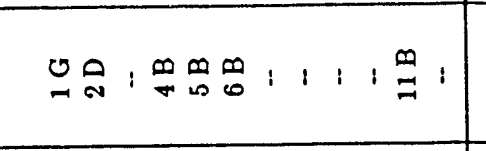 & 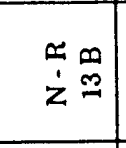 & 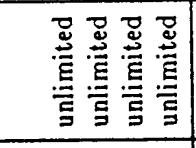 & 음욤 & 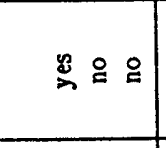 & $\stackrel{\circ}{\circ}$ & $\stackrel{\Delta}{2}$ & 우 \\
\hline$\approx$ & 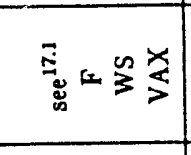 & 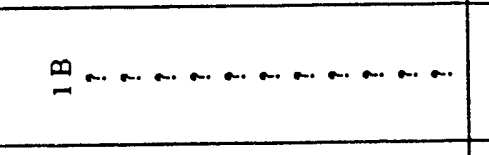 & Фّ̊ & 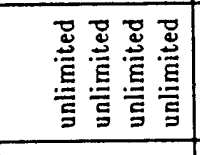 & $\ldots \ldots g$ & $\stackrel{8}{\infty} \cdots$ & $\stackrel{0}{2}$ & 욤 & |: \\
\hline $\mathscr{1}$ & 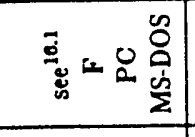 & 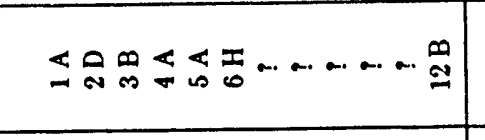 & $\ldots$ & 융요 \& & 용요욤 & $\cdots \cdots$ & 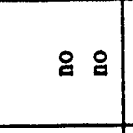 & $\stackrel{2}{\circ}$ & $\underset{8}{:}$ \\
\hline 2 & $1 \ldots \ldots$ & 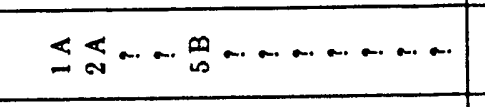 & $\sum^{\infty}$ & $\ldots \ldots \ldots$ & 론ㅇㅇㅇㅇㅇㅁㅇ & 옥음 & 욤욤 & 욤 & $\cdots$ \\
\hline$\Xi$ & 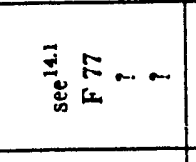 & 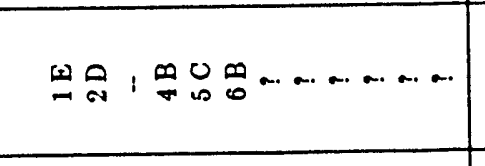 & 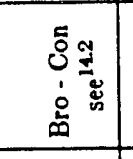 & 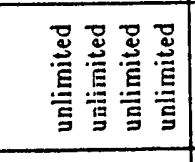 & 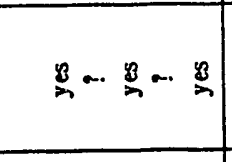 & $\stackrel{8}{2}$ & 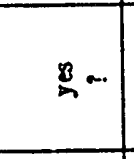 & $\mathscr{8}$ & 인 \\
\hline$m$ & 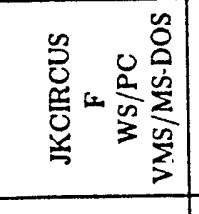 & 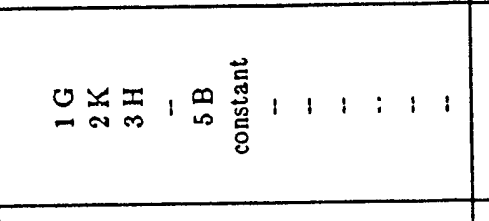 & 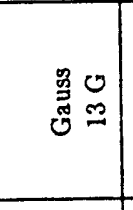 & 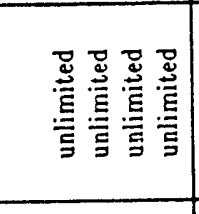 & ㅇㅇㅇㅇ & $\stackrel{\mathscr{2}}{2} \stackrel{\circ}{2}$ & 음 & 욤 & $\stackrel{2}{21}$ \\
\hline$\approx$ & 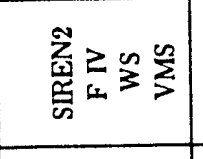 & 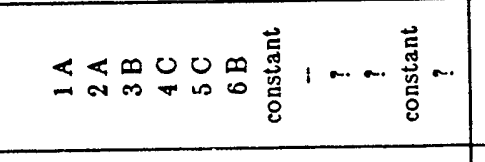 & 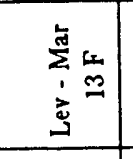 & 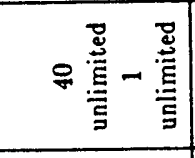 & 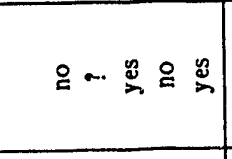 & $\stackrel{丶}{\check{n}} \circ$ & $\stackrel{9}{2}$ & $\stackrel{\circ}{\searrow}$ & 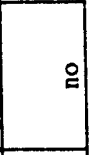 \\
\hline 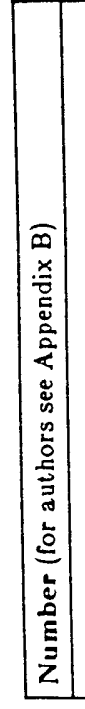 & 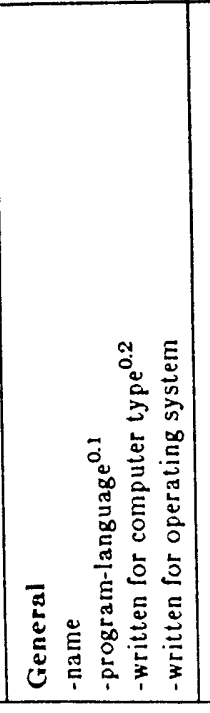 & 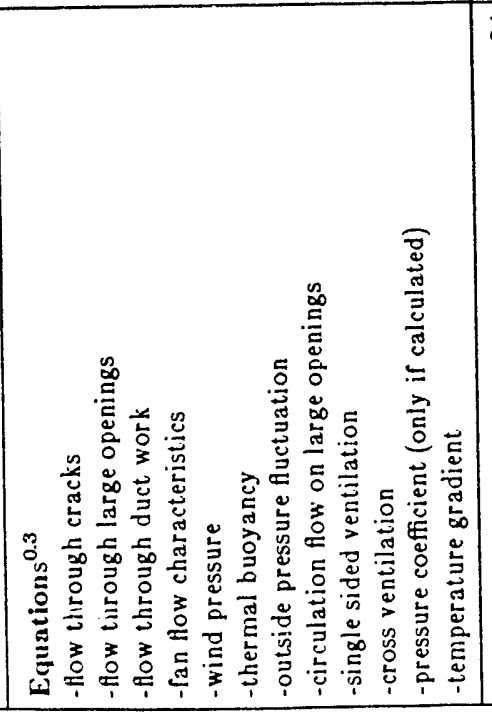 & 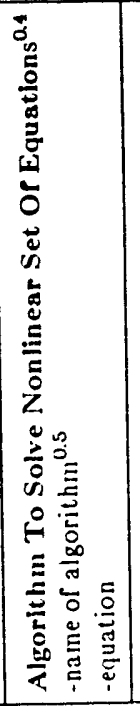 & 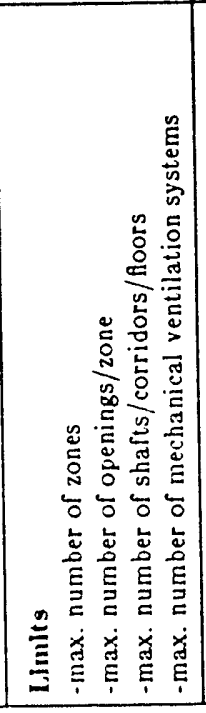 & 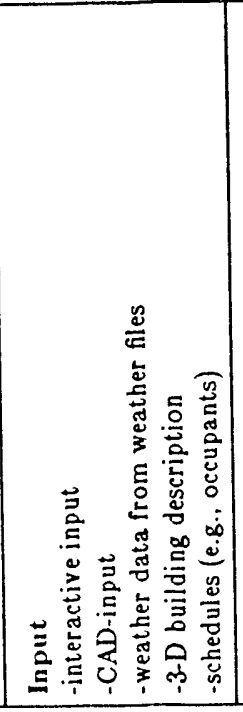 & 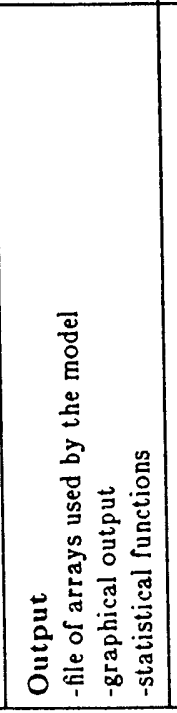 & 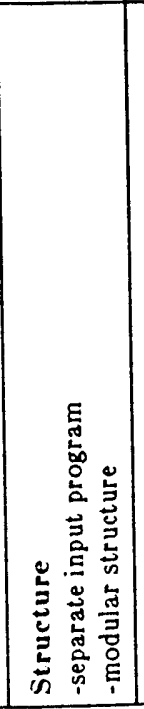 & 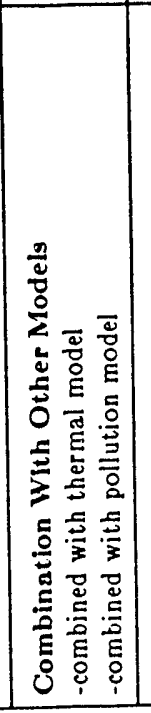 & 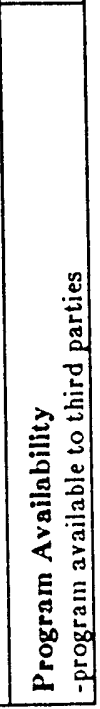 \\
\hline
\end{tabular}




\begin{tabular}{|c|c|c|c|c|c|c|c|c|c|}
\hline $\overrightarrow{g_{0}}$ & 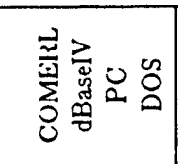 & « & 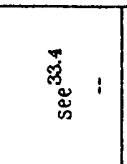 & 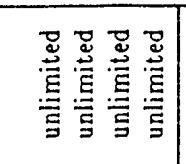 & 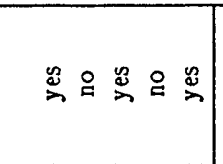 & 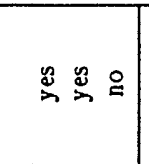 & $\stackrel{\mathscr{B}}{\triangle}$ & 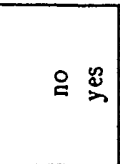 & 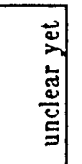 \\
\hline$\tilde{ల}$ & 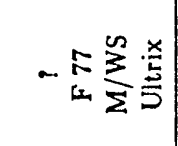 & 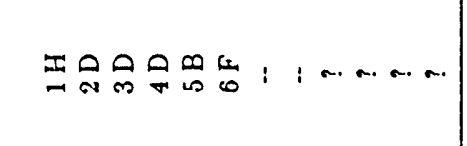 & 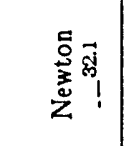 & 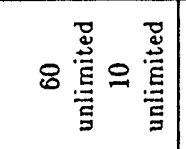 & $a \ldots a r$ & $\cdots \cdots$ & $\cdots$ & $\ldots \ldots$ & - \\
\hline$\vec{~}$ & 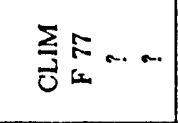 & 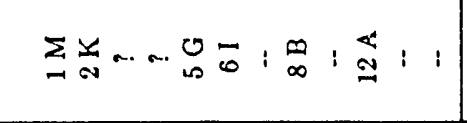 & 离 & 모요 & $\cdots \cdots$ & 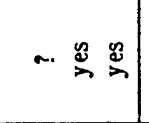 & 음욤 & $\stackrel{\sharp}{2}$ & 인 \\
\hline D & 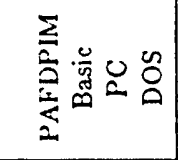 & 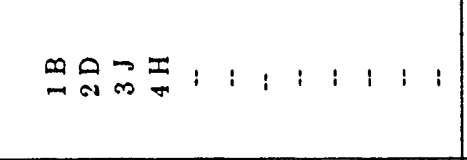 & 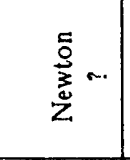 & $\sigma N: N$ & ฏ̊ㅇㅇㅇ & 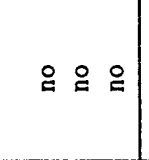 & $\stackrel{\circ}{2}$ & 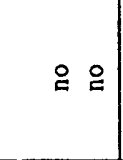 & 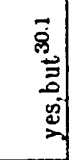 \\
\hline : & 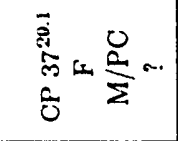 & 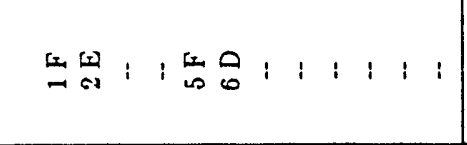 & 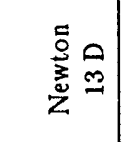 & $\stackrel{2}{\alpha}+\infty a$ & 용요 & $\stackrel{丶}{2} \stackrel{\circ}{\circ}$ & $\stackrel{\square}{\square}$ & $\stackrel{\circ}{\circ}$ & $\stackrel{\infty}{\infty}$ \\
\hline 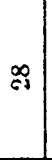 & 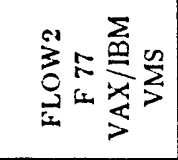 & 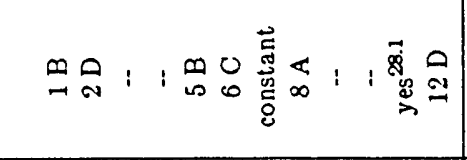 & 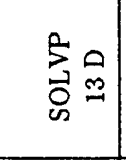 & 总总总总 & 욤 名 & 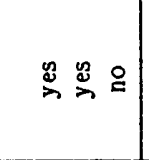 & $\stackrel{\Xi}{\varpi}$ & 岕 & 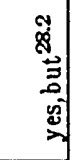 \\
\hline$\therefore$ & $\cdots \Phi \Sigma a$ & 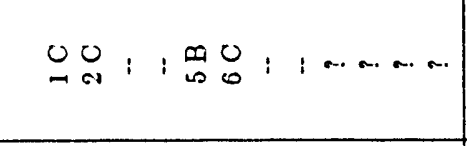 & 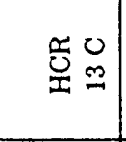 & 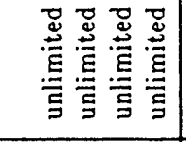 & 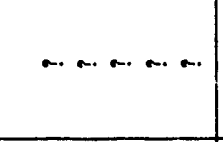 & $a \cdots$ & $\cdots$ & $\cdots$ & $\stackrel{\mathscr{2} 2}{2}$ \\
\hline \& & 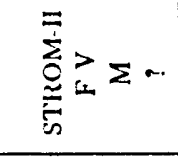 & 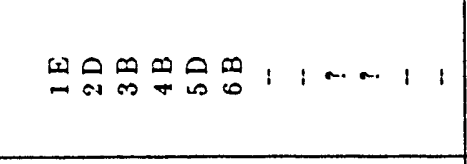 & 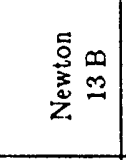 & 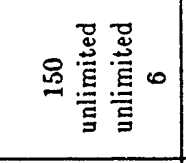 & $\stackrel{丶}{二}$ & $\stackrel{\infty}{2} \circ \stackrel{\circ}{\circ}$ & 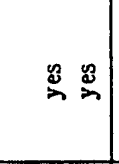 & 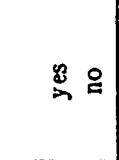 & 운 \\
\hline$\because$ & 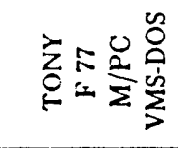 & 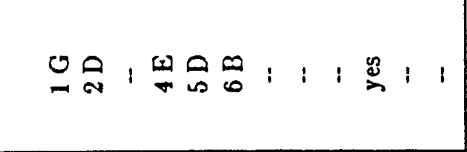 & 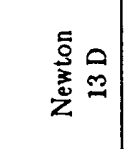 & 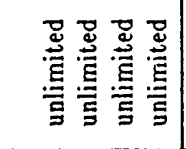 & 욤욤 & $\stackrel{\circ}{\circ} \circ$ & $\mathscr{2}$ & $\stackrel{丶}{\square}$ & 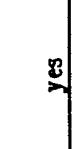 \\
\hline$\pi$ & 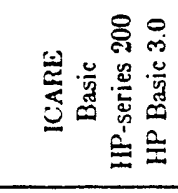 & 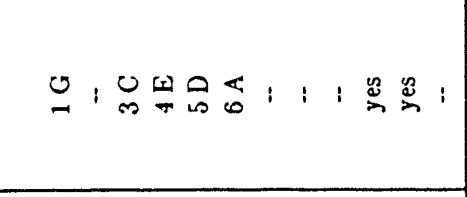 & 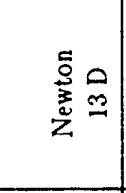 & W & 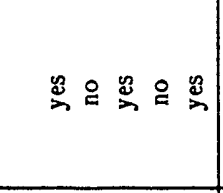 & 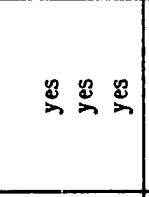 & $\stackrel{g}{2}$ & 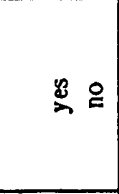 & 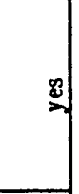 \\
\hline$\approx$ & 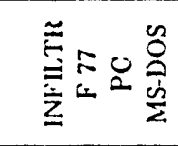 & 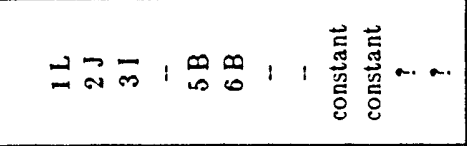 & $\cdots \frac{\infty}{m}$ & 恿 & 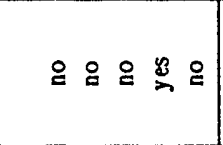 & 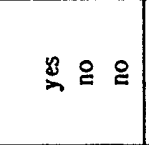 & $\nsubseteq$ & 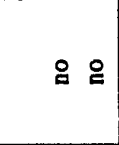 & $\sim$ \\
\hline 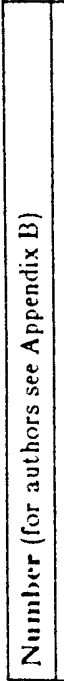 & 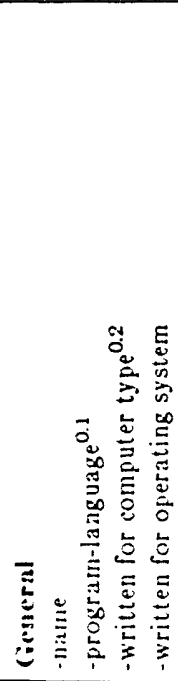 & 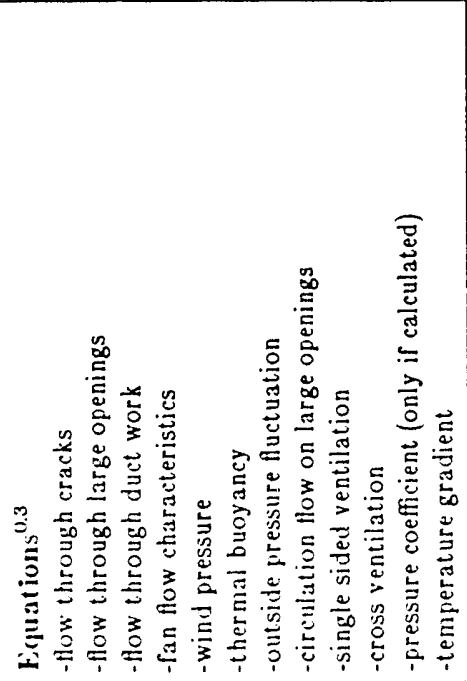 & 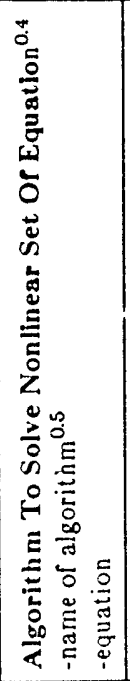 & $\begin{array}{l}r \\
\end{array}$ & 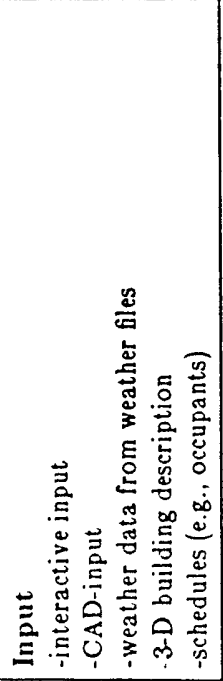 & 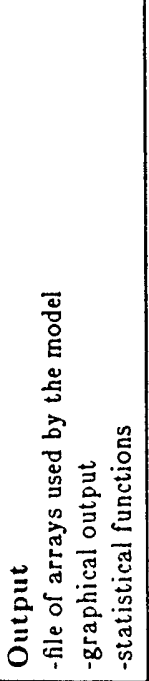 & 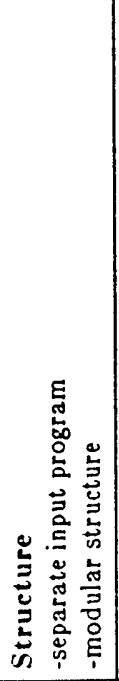 & 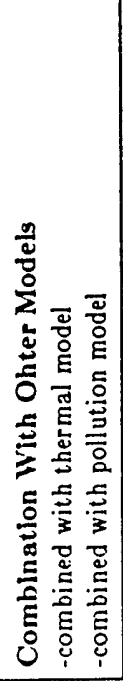 & 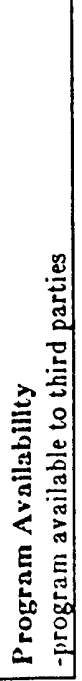 \\
\hline
\end{tabular}




\begin{tabular}{|c|c|c|c|c|c|c|c|c|c|}
\hline$\mp$ & $\cdots=\frac{\stackrel{5}{g}}{\frac{0}{0}} \sim$ & 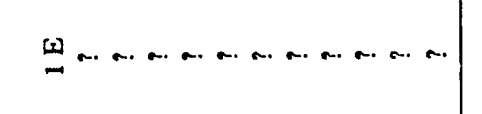 & $\cdots$ & 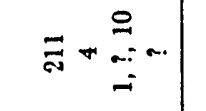 & $\ldots \ldots$ & $a \cdots$ & $\cdots$ & $\cdots a$ & 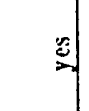 \\
\hline$F$ & 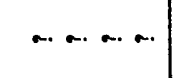 & $\nsubseteq \ldots \ldots \ldots \ldots \ldots \ldots \ldots \ldots \ldots$ & $\cdots$ & $\ldots \ldots \ldots$ & $\ldots \ldots \ldots \ldots$ & $\ldots \ldots$ & $\cdots a$ & $\cdots$ & . \\
\hline ₹ & $\ldots \ldots \ldots$ & 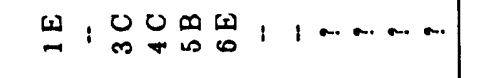 & $\cdots$ & $\cdots+\infty$ & $\cdots \cdots \cdots$ & $\ldots \ldots$ & $\cdots$ & $\cdots \cdots$ & - \\
\hline $\bar{F}$ & 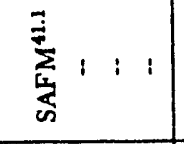 & 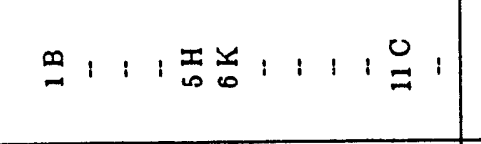 & $1:$ & 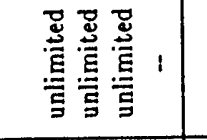 & ㅇㅇㅇㅇㅛ & 읃 & 응 & $\cong:$ & 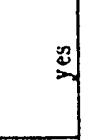 \\
\hline F & 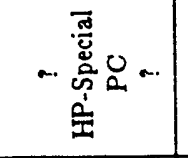 & 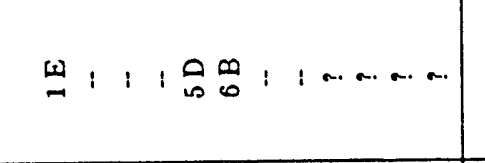 & $: \overrightarrow{\vec{q}}$ & 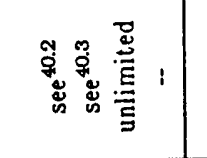 & 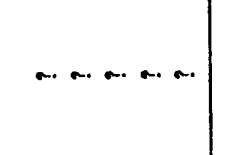 & $\cdots \cdots$ & $\cdots$ & $\cdots$ & $\stackrel{c}{a}$ \\
\hline g & 一全狊品。 & 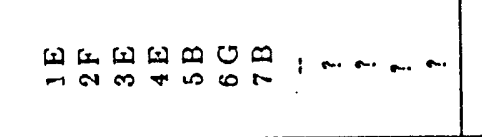 & 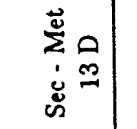 & 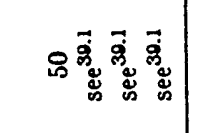 & $\ldots \ldots \ldots$ & $\ldots \ldots$ & $\cdots$ & $\ldots$ & 이 \\
\hline$\infty$ & 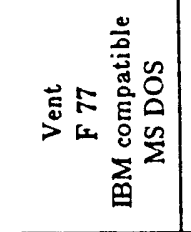 & 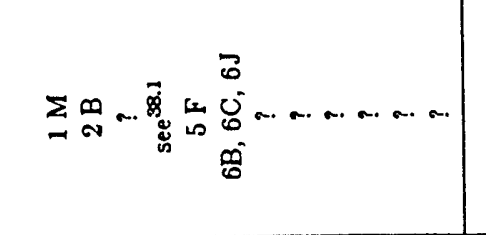 & 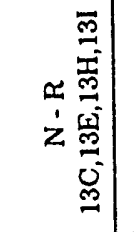 & \&్ర & 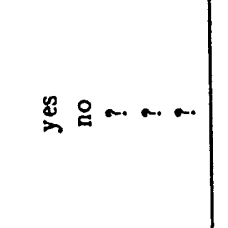 & $\mathscr{B} \ldots \ldots$ & 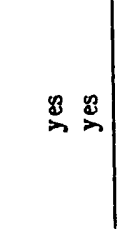 & $\stackrel{8}{\infty}$ & 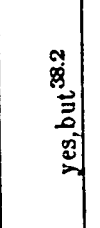 \\
\hline m. & $\ldots \geq \Sigma \Sigma$ & 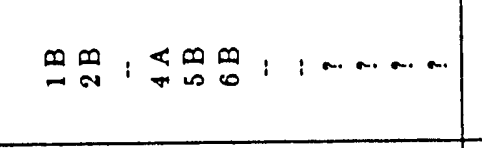 & 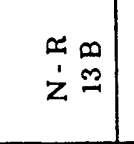 & 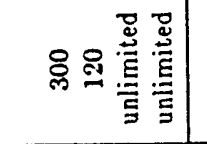 & 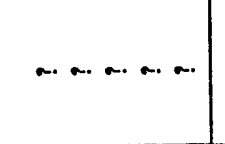 & $\cdots \infty$ & $\ldots$ & $\ldots$ & |ֶ̆: \\
\hline$\stackrel{\oplus}{\infty}$ & 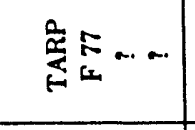 & 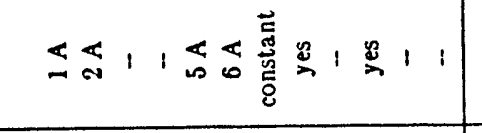 & 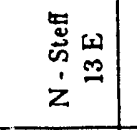 & 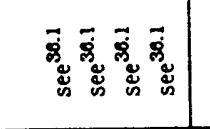 & 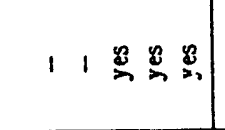 & $1: 11$ & $\stackrel{2}{2}$ & 82 & 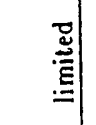 \\
\hline$\infty$ & 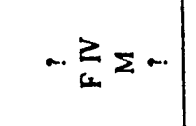 & 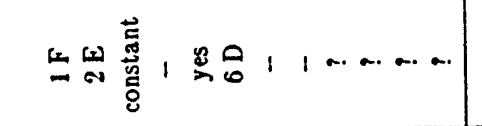 & $\cdots \stackrel{0}{m}$ & $\mathscr{q}+-\infty$ & $\cdots$ & $a \cdots$ & $\cdots$ & $\cdots$ & $\stackrel{2}{2}$ \\
\hline $\bar{m}$ & 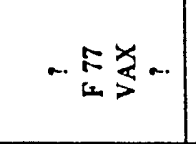 & 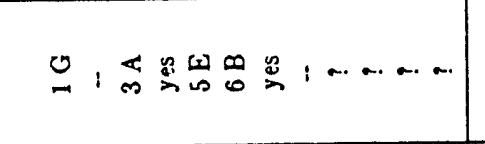 & 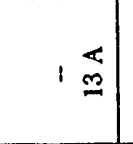 & 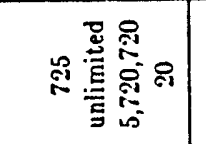 & $\cdots \cdots$ & $\cdots \cdots$ & $\cdots$ & $\cdots$ & 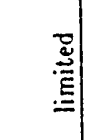 \\
\hline 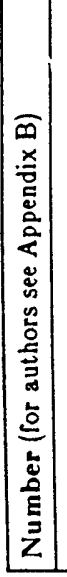 & 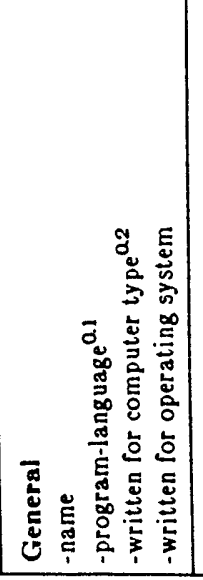 & 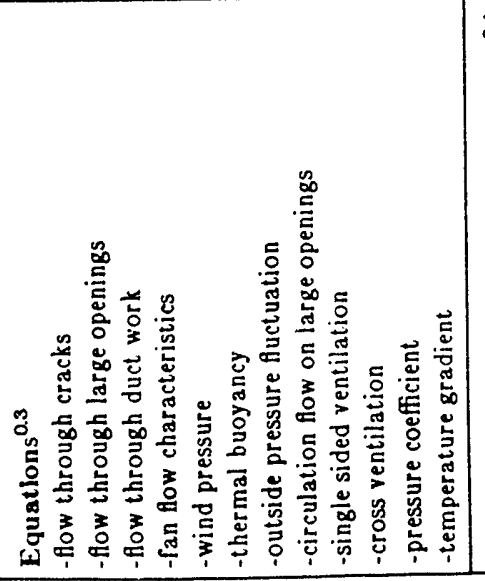 & 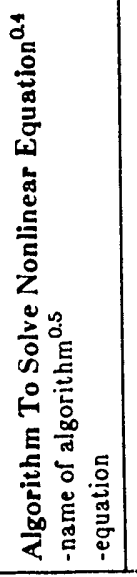 & 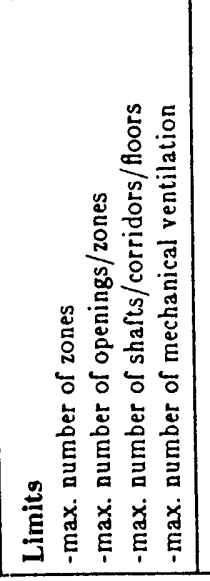 & 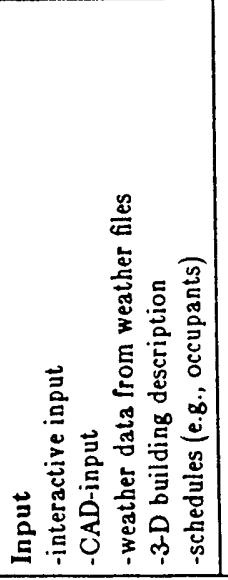 & 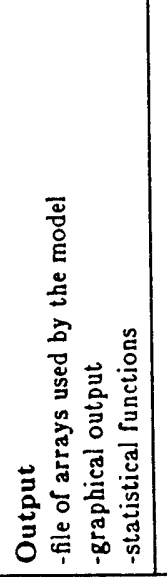 & 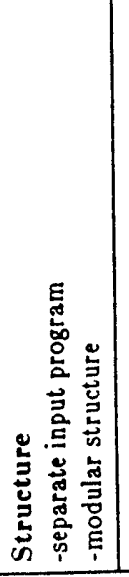 & 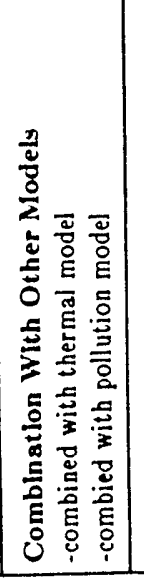 & $\begin{array}{ll} \\
\end{array}$ \\
\hline
\end{tabular}




\begin{tabular}{|c|c|c|c|c|c|c|c|c|c|}
\hline 8 & 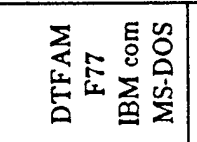 & 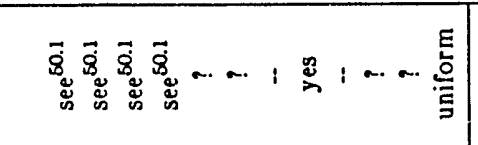 & $\dot{z}^{\alpha}$ & 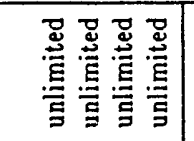 & 용⿱口口心 & $\stackrel{g}{2}:$ & 욤 & $\stackrel{\circ}{\square}$ & : \\
\hline of & 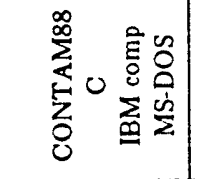 & 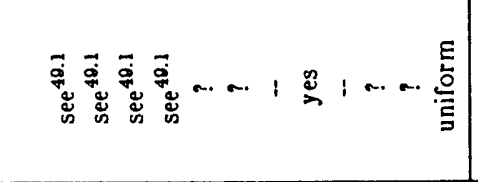 & $\stackrel{2}{z}$ & 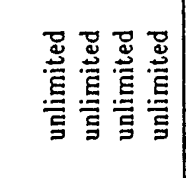 & 음 & 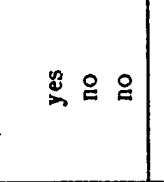 & $\stackrel{g}{ \pm}$ & 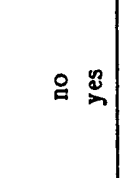 & 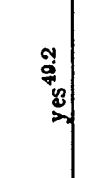 \\
\hline 必 & 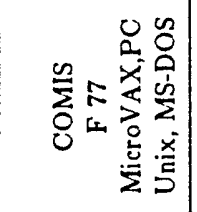 & 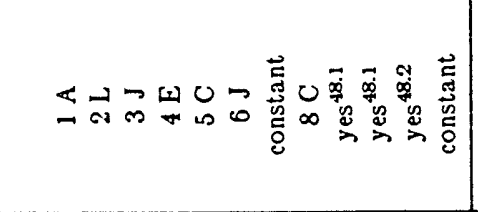 & 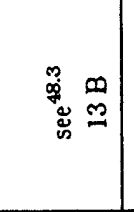 & 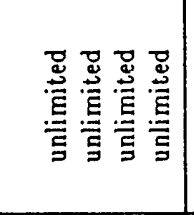 & 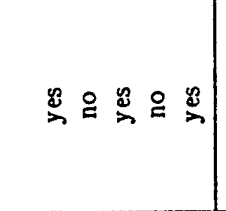 & $\stackrel{g}{2} \stackrel{0}{2}$ & $\stackrel{g}{ \pm}$ & $\stackrel{8}{\stackrel{\mathscr{B}}{\sim}}$ & 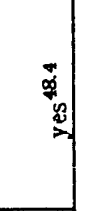 \\
\hline$F$ & 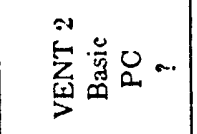 & 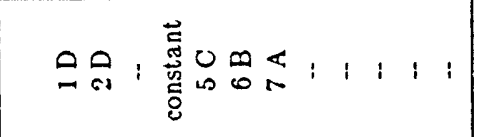 & $\overrightarrow{\mathbf{S}_{\mathrm{W}}}$ & 융 & 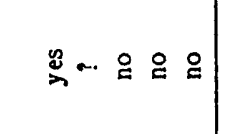 & 용욤 & 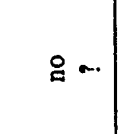 & $\stackrel{\circ}{\square}$ & 密 \\
\hline$\%$ & 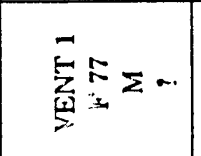 & 응: & 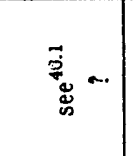 & 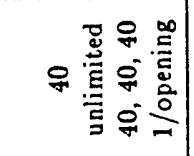 & 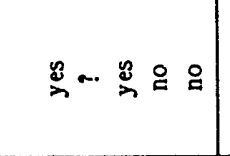 & 욤욤욤 & $\stackrel{ \pm}{\infty} \cdots$ & $\stackrel{8}{2}$ & 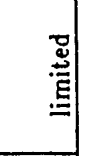 \\
\hline 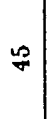 & 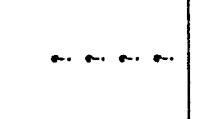 & 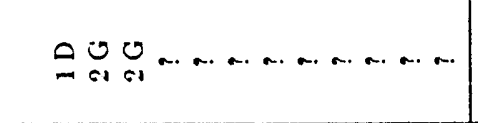 & 竞 & 员 …… & $a \ldots a r a$ & $\ldots \ldots$ & $\ldots$ & $\cdots$ & $\cdots$ \\
\hline 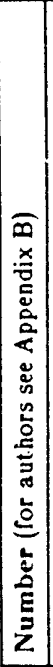 & 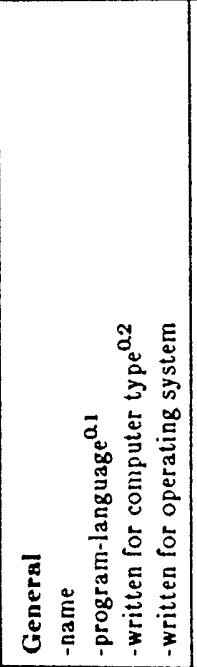 & 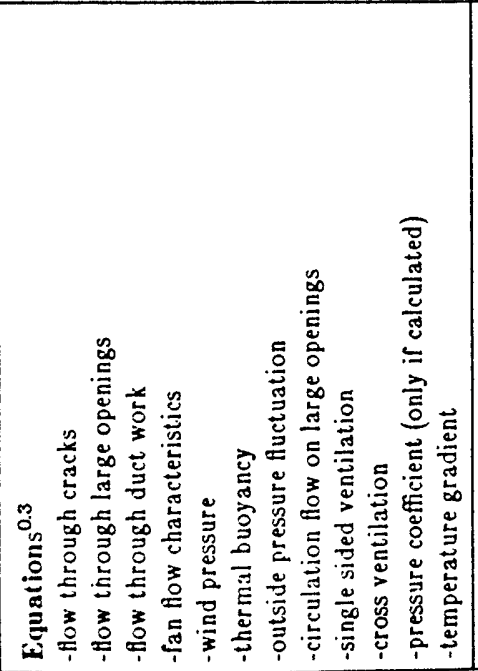 & 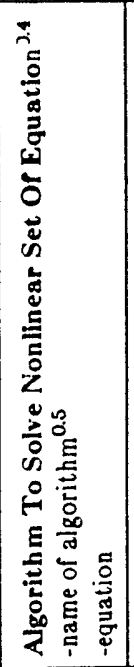 & 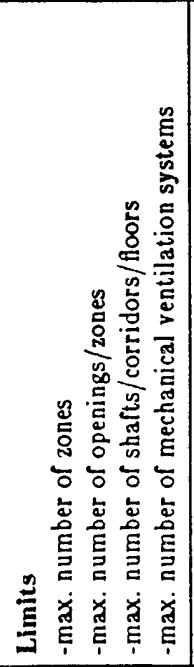 & 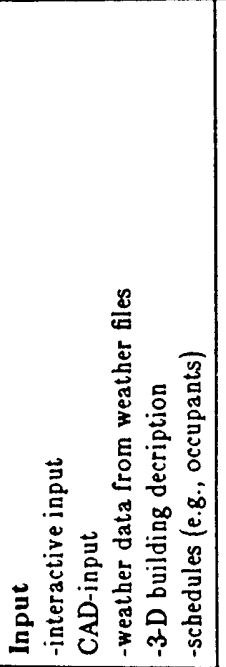 & 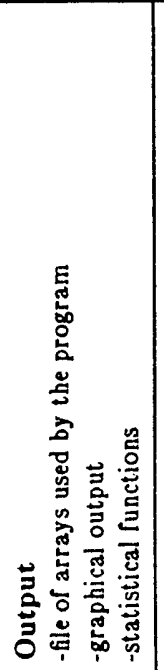 & 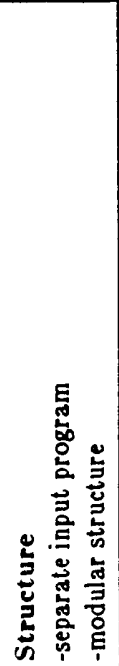 & 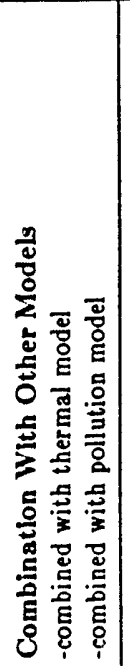 & 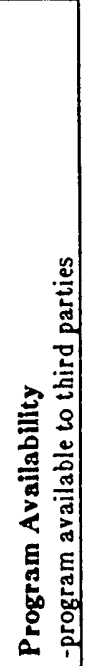 \\
\hline
\end{tabular}


Notes used in Appendix A

0.1) Abbreviations used for the Computer Language:

\begin{tabular}{ll} 
F & \multicolumn{1}{c}{ Fortran } \\
F 77 & Fortran 77 \\
MS & MicroSoft \\
F IV & Fortran IV \\
C & program language "C" \\
HP Basic 4.0 & Hewllett Packard Basic 4.0 \\
F V & Fortran V \\
dBaseIV & Data Base Language \\
HPL & Hewllett Packard Language \\
HP-Special & Hewllett Packard Special
\end{tabular}

0.2) Abbreviations used for the Computer Type:

PC Personal Computer

M Mainframe

WS Workstation

VAX Vertual Address Extension

IBM International Business Machine

0.3) for Equations see Table 1 - 12, Appendix C

0.4) for Algorithm to Solve Nonlinear Set of Equation see Table 13, Appendix C

0.5) Abbreviations used for the Name of Algorithm to Solve Nonlinear Set of Equation:

$N-R$

Newton - Raphson - Method

MNR

Modified Newton - Raphson Method

Lev - Mar Levenberg - Marquardt Method

Gauss Gaussian Elimination

Bro - Con

Brown - Conte Method

Beta

Beta Method by New mark

N-Steff Newton Method with Steffensen Acceleration

Sec-Met Secant Method

1.1) Kajima General Ventilation Calculation Program

2.1) Calculation of Infiltration

2.2) under consideration

2.3) not so useful

4.1) Passive System Simulation Program / Multiroom Version 1

4.2) nut in particular

4.3) integration of $1 \mathrm{E}$ or $2 \mathrm{D}$

4.4) Transformed Newton Method for the System of Nonlinear Equations using Jacobian Matrix

4.5) under the revise for new version

5.1) still under development

5.2) by Dividing Them to Horizontal Equidistant Strips

5.3) Lineare Interpolation from CPBANK 
5.4) Internal Reference Pressure Adjusted until Flow Errors Negligible

5.5) Not Fully Tested

5.6) Optional

5.7) after completing and testing

7.1) Infiltration and Ventilation

8.1) AIR-Calculation

9.1) Numerically Derived Jacobian-Matrix is inversed and Calculated Pressure Correction Vector is multiplied by 0.5 for Avoiding Oscillation

9.2) only in Japanese

10.1) Reference Pressures adjusted until Flow Errors Negligible

14.1) Simultaneous Heat, Moisture And Ventilation Predicting Program Multi Rooms

14.2) revised Newton - Raphson Method

16.1) Modified - Walton (NBS) Algorithm

16.2) not yet

16.3) not yet (used at users risk)

17.1) Model For Fluctuating Wind Pressure

19.1) alternate

20.1) Lineare interpolation between given points

20.2) Method with optimized Acceleration

22.1) Secant and $u / L$ - Decomposition

28.1) Harmonic Analysis (Reference 1) and Lagrange Interpolation (Reference 2)

28.2) with some restrictions

29.1) Fortran Program to Calculate Air Infiltration in Buildings - May 1974

29.2) but a second program, named CP 46 , is combined with pollution model (smoke concentration)

30.1) its applicability is very limited

32.1) Iterative technique using linear approximation to set of nonlinear equations including $Q$ and $P$.

33.1) still under development

33.2) treated like large openings

33.3) calculated by CPCALC, calculation program, see "Fundamentals of the Multizone", Air Infiltration and Ventialtion Centre, Technical Note 29, May 1990

33.4) optimized Newton, Newton with given relaxation, Newton-Steffensen

36.1) It is intended that the program be compiled with the appropriate values of a few parameter statements to match the program to the problem.

38.1) still under development

38.2) still under development

39.1) total number of connections smaller than 200

40.1) The exact nonlinear equations were solved to determine the volume flow rate. The balance of in- and outflowing air was determined by itcration (variation of the neutral pressure level NPL). 
40.2) In horizontal direction (per floor): 3 groups of rooms; outside group divided in windward and leeward flats, inner group (shafts), middle group (corridors) combine the different groups. In vertical direction: 30

40.3) one to each attached region

41.1) Simplified Air Flow Model

46.1) Internal reference pressure adjusted until flow errors negligible

47.1) Internal references pressure adjusted until flow errors negligible

48.1) treated like large openings

48.2) calculated by CPCALC, calculation program, see "Fundamentals of the Multizone", Air Infiltration and Ventilation Centre, Technical Note 29, May 1990

48.3) optimized Newton, Newton with given relaxation, Newton-Steffenson

48.4) as soon as fully debugged

49.1) Equation to be specified by the user

49.2) available at the National Institute of Standards and Technology

50.1) Equation to be specified by the user

50.2) available at the National Institute of Standards and Technology 


\section{APPENDIX B}

The authors and references of the computer programs

\begin{tabular}{|c|c|}
\hline Model - Number : & 1 \\
\hline Author: & $\begin{array}{l}\text { Shin Hayakawa } \\
\text { Building Environmental Engineering Department } \\
\text { Kajima Institute of Construction Technology } \\
\text { Kajima Corporation } \\
2-19-21 \\
\text { Chofu-City, Tokyo } \\
\text { Japan }\end{array}$ \\
\hline References : & $\begin{array}{l}\text { 1. Hayakawa, S.; Togari, S.; Hioki, M.: "Air Flow Rate Variation of HVAC } \\
\text { caused by Stack Effect and Opening a Window"; Third International Symposium } \\
\text { on the Use of Computers for Environmental Engineering related to Building, pp } \\
627-636, \text { Banff, Canada, Mai } 1978\end{array}$ \\
\hline Model - Number : & 2 \\
\hline Author: & $\begin{array}{l}\text { Hitoshi Yamazaki } \\
\text { Department of Architecture } \\
\text { Faculty of Engineering } \\
\text { Oita University } \\
700, \text { Dan-no-haru } \\
\text { Oita-shi, 870-11 } \\
\text { Japan }\end{array}$ \\
\hline References: & - \\
\hline Model - Number: & 3 \\
\hline Author: & $\begin{array}{l}\text { Mitsuhiro Udagawa } \\
\text { Department of Architecture } \\
\text { Kogakuin University } \\
\text { 1-24-2. Nishi-Shinjyuku } \\
\text { Shinjyuku-ku, Tokyo } \\
\text { Japan, } 160\end{array}$ \\
\hline References & $\begin{array}{l}\text { 1.Ishida. K.-I.: Udagawa, M.:"A Practical Method for Calculation of Room Tem- } \\
\text { perature Variation Considering Natural Ventilation and Radiant Heat Exchange } \\
\text { among Room Surfaces in Multi - Room Buildings"; Transactions of the Architec- } \\
\text { tural Institute of Japan, Nov. } 1987\end{array}$ \\
\hline & $\begin{array}{l}\text { 2. Ishida, K.-I.; Cdagawa, M.: "Validation of the Ventilation Net Work Model for } \\
\text { the Estimation of Room Temperatures and Ileat Load of Residential Buildings } \\
\text { with Measured Data"; Traisaction of the Architectural Institute of Japan, Oct. } \\
1988\end{array}$ \\
\hline & $\begin{array}{l}\text { 3. Ishida, K.-I.; Udagawa, M.: "Ventilation of Simulation Program for the Esti- } \\
\text { mation of Thermal Performance of Residential Houses using the Data from the } \\
\text { Year Round Monitoring"; CIIMA 2000, } 1985\end{array}$ \\
\hline
\end{tabular}




\begin{tabular}{|c|c|}
\hline Model - Number : & 4 \\
\hline Author: & $\begin{array}{l}\text { Tetsuo Hayashi } \\
\text { Department of Thermal Energy System } \\
\text { Interdisciplinary Graduate School of } \\
\text { Engineering Sciences, Kyushu University } \\
6 \text { - } 1 \text { Kasuga-Kouen } \\
\text { Kasuga-Shi, Fukuoka-Ken, } 816 \\
\text { Japan }\end{array}$ \\
\hline References : & $\begin{array}{l}\text { 1. Hayashi, T.; Urano, Y.; Watanabe, T.; Ry } \\
\text { Program "PSSP" and its Applications", Proc } \\
\text { tion Conference 1985, Seattle, USA } \\
\text { 2. Hayashi, T.; Urano, Y.; Katayama, T.; Suga } \\
\text { Ryu, Y.: "Prediction of Air Distribution in M } \\
\text { the Roomvent-87 (Air Distribution in Ventilat }\end{array}$ \\
\hline Model - Number : & 5 \\
\hline Author: & $\begin{array}{l}\text { Karoly Balazs } \\
\text { Hungarian Institute for Building Science (eti) } \\
\text { P.O.B. } 71 \\
\text { XI. David F. u. } 6 \text {. } \\
\text { Budapest } 1113 \text {, Hungary }\end{array}$ \\
\hline References: & -- \\
\hline Model - Number : & 6 \\
\hline Author: & $\begin{array}{l}\text { Ian C. Ward } \\
\text { University of Sheffield } \\
\text { Dept. cf Bldg. Science } \\
\text { Sheffield S10 2TN } \\
\text { United Kingdom }\end{array}$ \\
\hline References: & -- \\
\hline
\end{tabular}




\begin{tabular}{|c|c|}
\hline $\begin{array}{l}\text { Model - Number : } \\
\text { Author : }\end{array}$ & $\begin{array}{l}7,8 \\
\text { Marian .B. Nantka } \\
\text { Institute of Heating, Ventilating and Air Protection } \\
\text { Silesian Technical University } \\
\text { Pstrowski 5, Room } 23 \\
44100 \text { Gliwice, Poland }\end{array}$ \\
\hline References : & $\begin{array}{l}\text { 1. "Effectiveness of Ventilation Functioning in Multi-Story Residential Houses"; } \\
\text { (in polish; District Heating, Heating \& Ventilation) No.6, pp 154,1981 } \\
\text { 2. "A Numerical Method for Air - Change Rate of Buildings Calculated"; Record- } \\
\text { ings of the } 5 \text { th International Cunference on HVAC, Czechoslovakia, Nov. 24-26, } \\
1981 \\
\text { 3. "Mathematical Method for Natural and Mechanical Air Flows Calculated" ( in } \\
\text { polish) }\end{array}$ \\
\hline $\begin{array}{l}\text { Model - Number : } \\
\text { Author : }\end{array}$ & $\begin{array}{l}9 \\
\text { Hiroyasu Okuyama } \\
\text { Division of Environmental Technique } \\
\text { Shimizu Corporation } \\
\text { Institute of Technology } \\
\text { 4-17-, Etchujima 3-chome, Koto-ku } \\
\text { Tokyo, } \\
\text { Japan }\end{array}$ \\
\hline References : & $\begin{array}{l}\text { 1. Okuyama, H. : "Theoretical Study on the Thermal Network Model in Build- } \\
\text { ings"; (Doctorate) Waseda University, Tokyo, Dec. } 1987 \\
\text { 2. Okuyama, H. : "Network Numerical Analysis Method for Heat Transfer and } \\
\text { Airflow in Buildings"; Proceedings of } 17 \text { th Symposium by the Committee of } \\
\text { Building Heat Transfer in Building Environment, Comitees of Architectural Insti- } \\
\text { tute of Japan, Aug. } 1987 \\
\text { 3. Okuyama, H. : "A Computer Modelling Method of Building Airflow Network } \\
\text { and The Solution of Non-linear Simultaneous Equations"; Annual Meeting } \\
\text { Proceedings of Society of Heating Air-Conditioning and Sanitary Engineers of } \\
\text { Japan, Oct. 1989, p } 729 \text {, (Japanese) }\end{array}$ \\
\hline Model - Number & 10 \\
\hline Author: & $\begin{array}{l}\text { Servando Alvarez Dominguez } \\
\text { Dpio. de Ingeneria } \\
\text { Energetica y Fluidomecanica } \\
\text { Escuela Tecnica Superior de } \\
\text { Ingenieros Industriales } \\
\text { Avda. Reina Mercedes } \\
\text { E-4101 Sevilla } \\
\text { Spain }\end{array}$ \\
\hline References: & -- \\
\hline
\end{tabular}




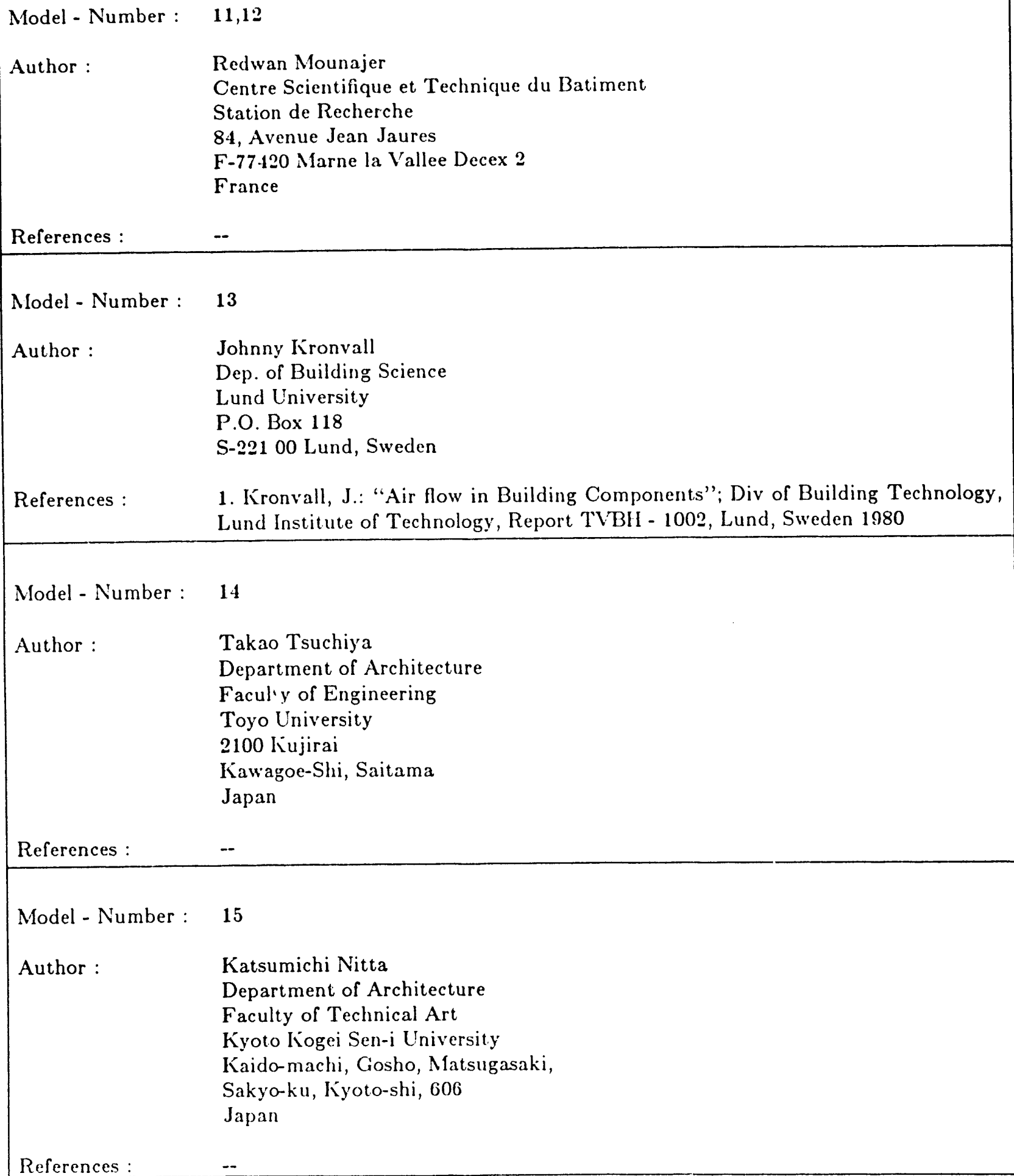




\begin{tabular}{|c|c|}
\hline Model - Number : & 16 \\
\hline Author : & $\begin{array}{l}\text { Martin Liddament } \\
\text { Air Infiltration and Ventilation Centre } \\
\text { University of Warwick Science Park } \\
\text { Barclays Venture Centre } \\
\text { Sir William Lyons Road } \\
\text { Coventry CVA 7EZ } \\
\text { Great Britain }\end{array}$ \\
\hline References: & -- \\
\hline Model - Number & 17 \\
\hline Author & $\begin{array}{l}\text { Takashi Sasaki } \\
\text { Department of Architecture } \\
\text { Hokkaido University } \\
\text { Nishi 8-chome, Kita 13-jou, Kitaku } \\
\text { Sapporo, } 006 \\
\text { Japan }\end{array}$ \\
\hline References: & $\begin{array}{l}\text { 1. Sasaki, T; Hayashi, M.; Aratani, N.: "On the Ventilating Characteristics of the } \\
\text { Space under the fluctuating Wind Pressure" Int. Conf. Roomvent 1987, Stock- } \\
\text { holm, Session ta, pp 1-12 }\end{array}$ \\
\hline & $\begin{array}{l}\text { 2. Sasaki, T; Aratani, N.: "Study on Change from Summer - Oriented Houses to } \\
\text { Winter - Oriented Ones and their Ventilation" Bulletin of Faculty of Engineering, } \\
\text { Hokkaido University No. 145, pp 125-151, Dec.1988 }\end{array}$ \\
\hline & $\begin{array}{l}\text { 3. Saski, T; et al: "Der Lueftungszustand von der Luftschicht durch die Wind- } \\
\text { Unruhe" Trans. AIJ (Architectural Institute of Japan) No. 372, Feb. } 1987\end{array}$ \\
\hline Model - Number : & 18 \\
\hline Author: & $\begin{array}{l}\text { Hiroshi Matsumoto } \\
\text { Toyohashi University of Technology } \\
\text { Department of Regional Planning } \\
\text { Tempakucho } \\
\text { Toyohashi-shi, } 440 \\
\text { Japan }\end{array}$ \\
\hline References & $\begin{array}{l}\text { 1. Matsumoto, H.; Nagatomo, M.; Yoshino, H.: "A Calculating Method for } \\
\text { Predicting Air Pollution in Multi-celled Buildings"; Meeting of Tohoku branch of } \\
\text { AIJ. 33-36, } 1988 \text { (in japanese) }\end{array}$ \\
\hline
\end{tabular}


Model - Number : 19

Author : $\quad$ George Walton

National Institute of Standards and Technology

BR/A 313

Washington D.C. 20234

United States of Amerika

References : $\quad$ 1. "AIRNET - a Computer-Program for Building Airflow Network Modeling"; National Institute of Standards Interagency Report NISIR 89-4072

2. Walton, G.N.: "Airflow Network Models for Element - Based Building Airflow Modeling"; ASHRAE Transactions, 1989.

Model - Number : 20

Author : Magnus Herrlin

Lawrence Berkeley Lab

Bldg. 90, Rm. 307.4

Berkeley, CA 94720

USA

References : $\quad$ 1. Herrlin, M.K.: "MOVECOMP : A Multizone Infiltration and Ventilation Simulation Program"; Air Infiltration Review, Volume 9, No. 3, May 1988

Model-Number : 21

Author: $\quad$ Yasuo Utsumi

Miyagi National College of Technology

48 Nodayama, Shiote, Medeshima

Natori 981-12

Japan

References : 
Model - Number : 22

Author: $\quad$ W. de Gids

TNO Division of Technology for Society

P.O. Box 217

2600 AE Delft

Netherlands

References :

Model - Number : $\quad \mathbf{2 3}$

Author: $\quad$ B. Spruit

ISSO - Netherlands

Institute for Study and Promotion of Research in the Field of Building Services

Postbus 20740

3001 JA Rotterdam

Netherlands

References: $\quad$ 1. "ISSO - 4" (to be published)

2. Liddament, M.: "Air Infiltration Calculation Techniques - An Application Guide"; Air Infiltration and Ventilation Centre, June 1986 
Model - Number : $\quad 2$

Author: $\quad$ F. Allard; J.J. Roux; D. Caccavelli

Laboratoire Equipement de l'Habitat

Centre de Thermique - URA CNRS 1372

INSA de Lyon

Batiment 307

F-69621 Villeurbanne Cedex

France

References: $\quad$ 1. Cacavelli, D.; Roux, J.J.; Roldan, A.: "Infiltration, Air Flow Distribution and Micro - Computation Roomvent 87, "Air Distribution in Ventilated Spaces"; Session $4 \mathrm{a}$, Stockholm 10/12 1987

2. Cacavelli, D.; Roux, J.J.; Allard, F.: "Transferts aerauliques dans les batiments"; presentation de deux niveaux d'approche ; Journee d'Etude de la Societe Francaise des Thermiciens, Etat des connaissances sur la ventilation, Paris 26 Nov. 87

3. Cacavelli, D.; Roux, J.J.; Allard, F.: "A Simplified Approach of Air Infiltration in Multizone Buildings"; 9th Air Infiltration and Ventilation Centre - Conference, Gent, Belgium, Vol 2 Sept. 1988

4. Cacavelli, D.; Roux, J.J.; Allard, F. : "Modelisation du comportement thermique des batiments multizones"; Adaptation a un processus de conception, These de Doctorat INSA de Lyon Nov. 88, $473 \mathrm{p}$

Model - Number : 25

Author: $\quad$ F. Allard; G. Achard; A. Roldan

Laboratoire Equipement de l'llabitat

Centre de Thermique - URA CNRS 1372

INSA de Lyon

Batiment 307

F-69621 Villeurbanne Cedex

France

References: $\quad$ 1. Roldan, A.; Achard, G.; Allard, F.: "Influence des infiltrations et des transferts aeraulique entre pieces sur la charge thermique d'un batiment multizone"; Third International Congress on Building Energy Management, ICBEM 87, Lausanne Sept. 87, pp $178-185$

2. Roldan, A.: "Etude thermique et aeraulique des envelopps de batiment". Influence des couplages interieurs et du multizonage; These de Doctorat INSA de Lyon, Dec. 1985, 310 p 
Model- Number : 26

Author: $\quad$ H. Esdorn; H.E. Feustel; J. Guo

Hermann-Rietschel-Institut

Technische Universitaet Berlin

Marchstrasse 4

D-1000 Berlin 10

Federal Republic of Germany

References: $\quad$ 1. Feustel, H.: "Beitrag zur theoretischen Beschreibung der Druck- und Luftmassenstromverteilung in natuerlichen und maschinell geluefteten Gebaeuden"; Dissertation Technische Universitaet Berlin, 1983, VDI-Fortschrittsberichte, Reihe 6, Nr. 151, VDI-Verlag, Duesseldorf

2. Guo, J. : "Weiterentwicklung des Programms gemaess 1) und 2) mit dem Ergebnis einer Beruecksichtigung thermischer Druckdifferenzen auch innerhalb eines Geschosses und einer spuerbaren Verringerung der Rechenzeiten"; Interner Institutsbericht

Model - Number : 27

Author: $\quad$ P. J. Jackman

Building Services Research

and Information Association

Old Bracknell Lane West

Bracknell, Berkshire RG12 4AH

United Kingdom

References : $\quad$ 1. Jackman, P.J.: "Heat Loss in Buildings as a Result of Infiltration"; BSE, Vol 42 (1974), pp 6-15

2. Jackman, P.J.: "A Study of the Natural Ventilation of tall Office Buildings"; JIIIVE, Vol 38 (1970), pp 103-118 
Model - Nuıber : 28

Author : $\quad$ Claudio Melo

Departmento de Engenharia Mecanica

Universidade Federal de Santa Catarina

CX. Postal 476

88.049 Florianopolis - SC

Brazil

References: $\quad$ 1. Allen, C.: "Wind Pressure Data Requirements for Air Infiltration Calculation" Air Infiltration and Ventilation Centre, Technical Note 13, Bracknell, UK, 1984

2. Bowen, A.J.: "A Wind Tunnel Investigation using Simple Building Models to Obtain Mean Surface Wind Pressure Coefficient for Air Infiltration Estimates"; National Aeronautical Establishment Report LTR LA 209, NRCC, Canada, 1976

Model - Number : 29

Author : $\quad$ Daniel M. Sander

National Research Council Canada

Institute for Research in Construction

Building M 24, Montral Road

Ottawa, Ontario

Canada K1A0R6

References :

Model - Number : $\quad 30$

Author : $\quad$ Krzysztof Klobut

Helsinki University of Technology

Faculty of Mechanical Engineering

Otakaari 4

SF - 02150 ESPOO

Finland

References : $-$

Model - Number : 31

Author : $\quad$ P. Dalicieux

Departement Applications de l'Electricite

77250 Moret sur Loing

France

References : 


\begin{tabular}{|c|c|}
\hline Model - Number : & 32 \\
\hline Author: & $\begin{array}{l}\text { Peter Warren } \\
\text { Building Research Establishment } \\
\text { Bucknalls Lane } \\
\text { Garston, Watford WD2 7JR } \\
\text { United Kingdom }\end{array}$ \\
\hline References: & $\begin{array}{l}\text { 1. Evers, E.; Waterhouse, A.: "A Computer Model for Analysing Smoke Move- } \\
\text { ment in Buildings"; Building Research Establishment CP } 69 / 78(1978)\end{array}$ \\
\hline Model - Number : & 33 \\
\hline Author: & $\begin{array}{l}\text { Viktor Dorer } \\
\text { EMPA ABT } 176 \\
\text { CH-8600 Duebendorf } \\
\text { Switzerland }\end{array}$ \\
\hline References: & -- \\
\hline Model - Number & 34 \\
\hline Author & $\begin{array}{l}\text { J. Gabrielsson } \\
\text { Ekono Association for Power and Fuel Economy } \\
\text { P.O. Box } 27 \\
00131 \text { Helsinki } 13 \\
\text { Finland }\end{array}$ \\
\hline References & $\begin{array}{l}\text { 1. Gabrielsson, J.; Porra, P.: "Calculation of Infiltration and Transmission Heat } \\
\text { Loss in Residential Buildings by Digital Computers" JHHE, Vol } 35 \text { (1968), pp } \\
357-368\end{array}$ \\
\hline
\end{tabular}




\begin{tabular}{|c|c|}
\hline Model - Number : & 35 \\
\hline Author: & $\begin{array}{l}\text { C. Y. Shaw } \\
\text { Institute for Research in Construction } \\
\text { National Research Council } \\
\text { Bldg. M-24, IRC, NRC, Montreal Road } \\
\text { Ottawa, Canada K1A OR6 }\end{array}$ \\
\hline References : & $\begin{array}{l}\text { 1. Sander, D.M.; Tamura, G.T.: "FORTRAN IV Program to Simulate Air Move- } \\
\text { ments in Multi-Storey Buildings"; DBR Computer Program No. 35, National } \\
\text { Research Council of Canada, (1974). }\end{array}$ \\
\hline & $\begin{array}{l}\text { 2. Sander, D.M.: "FORTRAN IV Program to Calculate Air Infiltration in Build- } \\
\text { ings"; DBR Computer Program No. 37, National Research Council of Canada } \\
(1974) \text {. }\end{array}$ \\
\hline & $\begin{array}{l}\text { 3. Shaw, C.Y.; Sander, D.M.; Tamura, G.T.: "A FORTRAN IV Program to } \\
\text { Simulate Stair-Shaft Pressurization Systems in Multi-Storey Buildings"; DBR } \\
\text { Computer Program No. 38, National Research Council of Canada (1974) }\end{array}$ \\
\hline Model - Number : & 36 \\
\hline Author : & $\begin{array}{l}\text { George Walton } \\
\text { National Bureau of Standards } \\
\text { BR/B } 114 \\
\text { Washington D.C. } 20234 \\
\text { United States of Amerika }\end{array}$ \\
\hline References : & $\begin{array}{l}\text { 1. Walton, G.N.: "Thermal Analysis Research Program" Reference Manual } \\
\text { NBSIR 83-2655, U.S. Department of Comerce, (1983) }\end{array}$ \\
\hline Model - Number : & 37 \\
\hline Author: & $\begin{array}{l}\text { Stephen Irving } \\
\text { Oscar Faber Consulting Engineers } \\
\text { Marlborough House } \\
\text { Upper Marlborough Road } \\
\text { St. Albans } \\
\text { Herts, AL1 3UT, Great Britain }\end{array}$ \\
\hline References: & $\begin{array}{l}\text { 1. Irving, S.J.; Wilson, A.P.: "Validation of a Smoke Movement Program"; Build- } \\
\text { ing Engineering Research \& Technology, Vol } 2 \text { (1984), No. 4, pp 151-159 } \\
\text { 2. Irving, S.J.: "The Computer Simulation of Smoke Movement during Building } \\
\text { Fires"; Fire Prevention Science and Technology, No. 22, (1979), pp 3-8 }\end{array}$ \\
\hline
\end{tabular}


Model Number : $\quad 38$

Author : Mike Holmes

Arup Research \& Development

13 Fitzroy Street

London IVIP 6BO

England

References: $\quad$ 1. Holmes, M. J.: "Design for Ventilation", 6th Air Infiltration and Ventilation Centre - Conference, September 16-19, 1985, Netherlands

2. Ilolmes, M. J.; Salusbury, T.: "Ventilation Design for a Bus Station", 9th Air Infiltration and Ventilation Centre - Conference held at Novotel Hotel, Gent, Belgium, 12-15 September 1988

Model - Number : $\quad 39$

Author :

W. de Gids

TNO Division of Technology for Society

P.O. Box 217

2600 AE Delft

Netherlands

References: $\quad$ 1. deGids, W.F.: "Calculation Method for the Natural Ventilation of Buildings"; Publication No. 632 of the TNO Research Institut for Environmental Hygiene, Delft, (1977)

Model-Number : $\quad 40$

Author: $\quad$ W. Brinkmann

Bundesbaudirektion Berlin

Fasanenstrasse 87

D - 1000 Berlin 12

Federal Republic of Germany

References: 1. Brinkmann, W.: "Zur Bestimmung des Lueftungswaermebedarfs hoher Gebaeude"; Dissertation. Technische Universitaet Berlin, 1980 
Model-Number: 41

Author: $\quad$ IIelmut E. Feustel

Lawrence Berkeley Laboratory

Building 90, Room 307.4

1 Cyclotron Rd

Berkeley, California 94720

USA

References: $\quad$ 1. Feustel, H.E.; Sherman, M.: "A Simplified Model for Predicting Air Flow in Multizone Structures" Energy and Buildings, Vol. 13, 1989, pp 217-230

Model - Number : $\quad 42$

Author: $\quad$ G. Hausladen

Schiedel GmbH \& Co

Lerchenstr. 9

Postfach 500505

D - Munich 50

Federal Republik of Germany

References : $\quad$ 1. Hausladen, G.: "Wohnungslueftung, Untersuchung der verschiedenen Lueftungsarten bzw. Lueftungspraktiken unter hygienischen, bauphysikalischen und energetischen Gesichtspunkten"; Dissertation Technische Universitaet Muenchen, 1980, Fortschrittsberichte der VDI Zeitschriften, Reihe 6, Nr. 73

2. Hausladen, G.: "Luftwechsel in Wohnungen"; Heizung, Lueftung Haustechnik, $1978, \mathrm{Nr} .1, \mathrm{pp} 21-28$

3. Krueger, W.; Hausladen, G.: "Einfluss von Wind- und thermischen Kraeften auf das Betriebsverhalten von mechanisch betriebenen Entlueftungsanlagen in mehergeschossigen Wohngebaeuden"; Forschungsbericht fuer Haustechnik, Technische Universitaet Muenchen, 1979 


\begin{tabular}{|ll} 
Model - Number : & 43 \\
Author : & $\begin{array}{l}\text { J. P. Cockroft } \\
\text { Building Services Research Unit } \\
\text { University of Glasgow } \\
\text { 3 Lillybank Gardens } \\
\text { Glasgow G12 8RZ } \\
\text { United Kingdom }\end{array}$ \\
References : & $\begin{array}{l}\text { 1. Cockroft, J.P.: "Air Flows in Buildings"; Proceedingsedings of the Environ- } \\
\text { ment Inside Buildings Symposium, Southend on Sea, 1979 }\end{array}$ \\
Model - Number : & $\begin{array}{l}\text { 2. Cockroft, J.P.: "Validation of Buildings and Systems Energy Prediction using } \\
\text { Real Measurements"; Computer Aided Design, Vol 14,1982, No. 1, pp 39-43 }\end{array}$ \\
Author : & $\begin{array}{l}\text { R. E. Billsborrow } \\
\text { Department of Building Science }\end{array}$ \\
Faculty of the Architectural Studies \\
University of Sheffield \\
Sheffield S10 2TN \\
United Kingdom
\end{tabular}


Model - Number :

Author :

References :

Model - Number :

Author :

References :

Reference for VENT 2 :
45

S. Swetlov Soviet Union

1. Swetlov, K.S.: "Calculations of Air Infiltration in Multi-Story Buildings using Electronic Computers"; Vodosnabize i Sanitarna Tekhinika, Vol 11, 1966, HVRA-Translation 122

$46, \cdot 17$

D. W. Etheridge

British Gas plc

Watson House

Peterborough Road

London SIV6 3HN

United Kingdom

1. Etheridge, D.W.; Alexander, D.K.: "The British Gas Multi Cell Model for Calculating Ventilation"; ASHRAE-Transactions, Vol 86, 1980, Part II, pp 808-821

2. Etheridge, D.W.: "Crack Flow Equations and Scale Effect"; Building and Environment, Vol 12, 1977, pp 181-189

3. Etheridge, D.W.; Nolan, J.A.: "Ventilation Measurements at Model Scale in a Turbulent Flow"; Building and Environment, Vol 1.1, 1979, pp 53-64

1. Etheridge, D.W.; Gale, R.: "Theoretical and Experimental Techniques for Ventilation Research in Buildings"; International Gas Conference, Paper C 18-33, London, June 1983. 
Model - Number : 48

Authors :

Francis Allard

Laboratoire Equipment de'l Habitat

Centre de Thermique

INSA de Lyon

Batiment 307

F-69621 Villeurbanne Cedex,

France

Helmut E. Feustel

Lawrence Berkeley Laboratory

Building 90, Room 307.t

Berkeley, CA 94720, USA

Eduardo Rodrigues Garcia

Dpt. Ingenieria Energetica y

Fluicio Mecanica

Escuela Superior de Ingenieros

Industriales

Avda. Reina Mercedes S/N

E-Sevilla, Spain

Maric Grosso

Dipartimento di Scienze e Techniche

Per i Processi di Insediamento

Politecnico Di Torino

Viale Mattioli 39

I-10125 Torino, Italy

Magnus Herrlin

Division for Building Services Engineering

Royal lnstitute of Technology

S-100.4 Stockholm

Sweden
Victor Dorer

EMPA ABT 175

CH-8600 Duebendorf

Switzerland

Liu Mingsheng

Mechanical Engineering Department

Texas A\&M University

College Station, TX 77840, USA

Hiroshi Yoshino

Dept. of Architecture

Faculty of Engineering

Tohoku University

Sendai, 980

Japan

Hans C. Phaff

MT-TNO

POB 217

2600 AE Delft

Netherlands

Yasuo Utsumi

Department of Architecture

Miyagi National College of Technology

Medeshima, Natori, Miyagi

Japan

References :

1. Feustel, H. E.; Raynor-Hoosen, A. (Editors): "Fundamentals of the Multizone Air Flow Model - COMIS", Air Infiltration and Ventilation Centre, Tech. Note TN 29, May 1990, Coventry, UK 
Model - Number : $\quad 49,50$

Author : James Axley

Bldg. Technical Program

3 - 437

Mass. Institute of Technology

77 Mass. Ave

Cambidge, MA 02139

USA

References :

1. Axley, J.; Grot, R.: "The Coupled Air Flow and Thermal Analysis Problem in Building Airflow System Simulation", ASHRAE Symposium on Calculation of Interzonal Heat and Mass Transport in Buildings, Vol. 95, Pt. 2: 1989.

2. Axley, J.; Grot, R.: "Coupled Airflow and Thermal Analysis for Building System Simulation by Element Assembly Techniques", Room Vent '90: Engineering Aero- and Thermodynamics of Ventilated Rooms (Submitted for publication 1/29/90): 1990 .

3. Grot, R.: "Users Manual - -NBSAVTS - CONTAM88", National Institute of Standards and Technology, 1990.

4. Walton, G.: "AIRNET - A Computer Programm for Building Airflow Network Modeling", NISTR 89-4072, National Institute of Standards and Technology, 1988. 


\section{APPENDIX C}

Equations used in the computer programs

Nomenclature see Table 14

TABLE 1: Equations used for flow through cracks

1 A) $\quad F=D \quad \Delta P^{D}$

1B) $Q=D \quad \Delta P^{\mathrm{D}}$

1C) $\frac{\mathrm{Q}}{3600}=\frac{\mathrm{D}}{3600} \Delta \mathrm{P}^{1 / \mathrm{n}}$

1 D) $\Delta \mathrm{P}=\mathrm{a} \mathrm{Q}^{2}+\mathrm{b} \mathrm{Q}$

1E) $Q=C L \Delta P^{\mathrm{a}}$

1 F) $\quad F=K \Delta P^{x}$

1G) $\quad \mathrm{Q}=\mathrm{C}$ A $\Delta \mathrm{P}^{\mathrm{a}}$

1H) $\Delta \mathrm{P}=\left(\frac{\mathrm{Q}}{\mathrm{KL}}\right)^{\mathrm{D}}$

1 I) $\Delta \mathrm{P}=0.5 \mathrm{~L} \rho \mathrm{Q}^{2}$

$1 \mathrm{~J}) \quad \Delta \mathrm{p}=\mathrm{a} F+\mathrm{b} \mathrm{F}^{2}$

1K) $\Delta p=s \frac{\rho}{2}\left(\frac{\mathrm{Q}}{\mathrm{A}}\right)^{\mathrm{n}}$

1L) $\quad F=\rho D \Delta p^{n} \quad$ with $n=\frac{2}{3}$

1M) $\quad \mathrm{Q}=\mathrm{K} \Delta \mathrm{p}^{\mathrm{n}}$ 
TABLE 2: Equations used for flow through large openings

2A) $\quad F=D \Delta P^{n}$

2B) $\quad Q=D \Delta P^{n}$

2 C) $\quad \frac{Q}{3600}=\frac{D}{3600} \Delta P^{1 / n}$

2 D) $\quad Q=A C\left(\frac{2 \Delta P}{\rho}\right)^{1 / 2}$

2E) $\quad F=K \Delta P^{x}$

2F) $\quad \mathrm{Q}=\mathrm{A}(2 \Delta \mathrm{P})^{1 / \mathrm{n}} \sqrt{(1 / \rho)}$

2 G) $\Delta P=S Q^{2}$

2H) $\Delta \mathrm{p}=\mathrm{a} F+\mathrm{b} \mathrm{F}^{2}$

2 l) $\Delta \mathrm{p}=\varsigma \frac{\rho}{2}\left(\frac{\mathrm{Q}}{\mathrm{A}}\right)^{\mathrm{n}}$

2J) $\quad \mathrm{F}=\rho \mathrm{D} \Delta \mathrm{p}^{\mathrm{n}} \quad$; with $\mathrm{n}=\frac{2}{3}$

2K) $\quad Q=K \Delta p^{n}$

2L) $\quad F=\frac{2}{3} w C_{d} \Theta \sqrt{\rho\left[2 \mathrm{~g}\left(\rho_{1}-\rho_{2}\right)-b_{\imath}\right]}|H-Z n|^{\frac{3}{2}}$ 
TABLE 3: Equations used for flow through duct work

3 A) $Q=C A\left[\mathrm{P}_{\mathrm{f}}-\rho_{\mathrm{f}} \mathrm{g}\left(\mathrm{H}_{\mathrm{r}}-\mathrm{H}_{\mathrm{f}}+\frac{\mathrm{H}_{\mathrm{i}}}{2}-\mathrm{H}_{\mathrm{s}}\right)-\mathrm{P}_{\mathrm{i}}+\rho_{\mathrm{i}} \mathrm{g}\left(\mathrm{H}_{\mathrm{s}}-\frac{\mathrm{H}_{\mathrm{i}}}{2}\right)\right]^{\mathrm{n}}$

3 B) $\quad Q=A \quad\left(\frac{2}{\rho}\right)^{1 / 2} \sum_{i=1}^{m}\left(\frac{1}{k}\right)_{j}^{1 / 2} \Delta p^{1 / 2}$

3C) $\quad \mathrm{P}-\frac{\rho}{2} \mathrm{v}^{2}(1+\Sigma \varsigma)+\Delta \mathrm{P}_{\mathrm{vE}}-\mathrm{P}_{\mathrm{D}}=0$

3D) $\Delta \mathrm{P}=\left[\mathrm{K}+\frac{\mathrm{f}_{\mathrm{L}}}{\mathrm{D}}\right] \frac{\mathrm{g}}{\mathrm{H}^{2} \mathrm{D}^{4} \rho} \mathrm{Q}^{2}$

3E) $Q=A_{\mathrm{NET}}(2 \Delta \mathrm{P})^{1 / \mathrm{n}}\left(\frac{1}{\rho}\right)^{1 / 2}\left(\frac{\lambda 1}{\mathrm{~d}}+\Sigma \varsigma\right)$

$3 F) \quad F=\frac{\left(\frac{2 \rho A^{2}}{f L / D+\Sigma C_{0}^{2}}\right)^{1 / 2}}{\Delta p}$

3G) $\quad Q=A\left(\frac{2 \Delta p}{\rho \varsigma}\right)^{(1 / n)}$

3H) $\quad \Delta \mathrm{p}=\varsigma \frac{1}{2} \rho \mathrm{v}^{2}$

$$
\text { and : } \Delta \mathrm{p}=\frac{\lambda \mathrm{l}}{\mathrm{d}} \frac{1}{2} \rho \mathrm{v}^{2}
$$

3 I) $\quad F=A\left(\frac{2 \rho \Delta \mathrm{p}_{\mathrm{K}}}{\left(\frac{\lambda \mathrm{I}}{\mathrm{d}}\right)+\Sigma \varsigma}\right)^{(1 / 2)}$

3J) $\Delta \mathrm{p}=\mathrm{C} \mathrm{Q}^{2}$ 
TABLE 4: Equations used for fan flow characteristics
4A) $Q=K_{1}+K_{2} \Delta P+K_{3} \Delta P^{2}$
4B) $\quad \Delta P=K_{1}+K_{2} Q+K_{3} Q^{2}$
4C) $\Delta P=\Delta P_{o}-K_{1} Q-K_{2} Q^{2}$
4D) $\Delta \mathrm{P}=\mathrm{B} \mathrm{n}^{2} \mathrm{D}^{2}+\frac{\mathrm{Q}^{2}}{\mathrm{~A}^{2} \mathrm{D}^{4}}$
4E) $\quad \Delta \mathrm{P}=\mathrm{f}\left(\mathrm{Q}_{v}\right)$
$4 \mathrm{~F}) \quad \Delta \mathrm{p}=\mathrm{K}_{0}+\mathrm{K}_{1} \mathrm{~F}+\mathrm{K}_{2} \mathrm{~F}^{2}+\mathrm{K}_{3} \mathrm{~F}^{3}$
4G) $\quad \Delta \mathrm{p}_{\mathrm{t}}=\mathrm{K}_{1}+\mathrm{K}_{2} \mathrm{Q}+\mathrm{K}_{3} \mathrm{Q}^{2}+\mathrm{K}_{4} \mathrm{Q}^{3}$
$4 \mathrm{H}) \quad \Delta \mathrm{p}=\mathrm{C}_{1}-\left(\mathrm{C}_{2} \mathrm{Q}^{2}\right)$

TABLE 5: Equations used for wind pressure
5 A) $\Delta \mathrm{P}=\frac{\rho}{2} \mathrm{v}^{2}$
5 B) $\Delta \mathrm{P}=\mathrm{C} \frac{\rho_{\mathrm{o}}}{2} \mathrm{v}^{2}$
5 C) $\Delta \mathrm{P}=\mathrm{C} \frac{\rho}{2} \mathrm{v}_{\mathrm{o}}^{2}$
5 D) $\Delta \mathrm{P}=\mathrm{C} \frac{\rho}{2} \mathrm{vI} \mathrm{I}^{2}$
5 E) $\Delta \mathrm{P}=\frac{\rho}{2} \mathrm{v}^{2}$
with: $\mathrm{v}=\mathrm{v}_{\mathrm{O}} 2334[1+2.8 \log (\mathrm{H}+4.75)]$

$5 \mathrm{~F}) \quad \Delta \mathrm{p}=\mathrm{K} \mathrm{v}^{2} \mathrm{cp} ;$ with : $\mathrm{cp}$ are input

$5 \mathrm{G}) \quad \mathrm{p}_{\mathrm{wind}}=\mathrm{cp} \frac{1}{2} \rho \mathrm{v}^{2}$

$5 \mathrm{H}) \quad \Delta \mathrm{p}=\mathrm{cp} \Delta \mathrm{p}_{\mathrm{d} y \mathrm{n}}$ 
TABLE 6: Equations used for thermal buoyancy
6 A) $\Delta \mathrm{P}=\rho \mathrm{g} \Delta \mathrm{II}$
6 B) $\Delta \mathrm{P}=\left(\rho_{\mathrm{o}}-\rho_{\mathrm{i}}\right) \mathrm{g}\left(\mathrm{H}-\mathrm{H}_{\mathrm{o}}\right)$
GC) $\Delta \mathrm{P}=3462\left(\mathrm{H}-\mathrm{H}_{\mathrm{o}}\right)\left(\frac{1}{\mathrm{~T}_{\mathrm{o}}}-\frac{1}{\mathrm{~T}_{\mathrm{i}}}\right)$
6 D) $\Delta \mathrm{P}=\left(\rho_{\mathrm{o}}-\rho_{\mathrm{i}}\right) \mathrm{gH}$
$6 \mathrm{E}) \quad \Delta \mathrm{P}=\left(\rho_{\mathrm{o}}-\rho_{\mathrm{i}}\right) \mathrm{gH}(\mathrm{X}-0.5-\mathrm{N} / \mathrm{2}) \mathrm{b}+\mathrm{c}$
$6 \mathrm{~F}) \quad \mathrm{Q}=\left(\mathrm{C}_{\mathrm{D}} \mathrm{A} / \mathrm{n}\right)\left(\Delta \Theta \mathrm{g} \frac{\mathrm{H}}{\bar{\theta}}\right)^{\mathrm{t} / 2}$
6 G) $\Delta P=\rho_{o}\left(\frac{T_{i}-T_{o}}{T_{i} T_{o}}\right) T_{o} g H$
$6 \mathrm{H}) \quad \Delta \mathrm{p}=\rho \mathrm{g} 273\left(\mathrm{~h}-\mathrm{h}_{0}\right)\left(\frac{1}{\mathrm{~T}_{0}}-\frac{\mathrm{t}}{\mathrm{T}_{\mathrm{i}}}\right)$
6 I) $\quad \Delta \mathrm{p}_{\mathrm{z}}=\Delta \rho \mathrm{gz}$
$6 \mathrm{~J}) \quad \Delta \mathrm{p}=\left(\mathrm{p}_{\mathrm{i}}-\rho_{\mathrm{i}} \mathrm{gh} \mathrm{h}_{\mathrm{i}}\right)-\left(\mathrm{p}_{\mathrm{j}}-\rho_{\mathrm{j}} \mathrm{g} \mathrm{h}_{\mathrm{j}}\right)$
6 K) $\Delta \mathrm{p}=\Delta \rho \mathrm{g} \Delta \mathrm{H}$

TABLE 7: Equations used for outside pressure fluctuations
$7 \mathrm{~A}) \quad\left(\Delta \overline{\mathrm{P}}^{2}\right)^{0.5}=0.5\left(\overline{\mathrm{P}}_{\mathrm{o}}{ }^{2}\right)^{0.5}$
7 B) $\quad Q_{v}=1 / 2 A_{\text {NET }}\left(0.001 v^{2}+0.0035 H \Delta T+0.01\right)^{1 / 2}$

TABLE 8: Equations used for circulation flow on large openings
8 A) $\quad Q_{x}=C_{d} A_{x}\left(\frac{2}{\rho} \Delta p\right)^{0.5}$
8 B) $\quad \mathrm{Q}=\mathrm{K} \Delta \mathrm{p}^{0.5} ;$ with : $\Delta \mathrm{p}=\Delta \rho \mathrm{gh}$
$8 \mathrm{C}) \Delta \mathrm{F}=\mathrm{F}_{\mathrm{O}, \mathrm{ZN}}-\mathrm{F}_{\mathrm{ZN}, \mathrm{H}}$

TABLE 9: Equations used for single sided ventilation

no equations were specified 
TABLE 10: Equations used for cross ventilation

$10 \mathrm{~A}) \quad \mathrm{Q}=\mathrm{K} \Delta \mathrm{p}^{0.67}$

TABLE 11: Equations used for pressure coefficient
$11 \mathrm{~A})$

$$
\begin{array}{lll}
\mathrm{cp}=0.75-1.05 \frac{\mathrm{Q}}{90} & \text { if } & \mathrm{Q} \leq 90^{\circ} \\
\mathrm{cp}=-0.45+0.15 \frac{\mathrm{Q}}{90} & \text { if } & \mathrm{Q}>90^{\circ} \\
\Rightarrow=>\quad \Delta \mathrm{p}_{\text {wind }}=\frac{1}{2} \rho \mathrm{cp} \mathrm{v}^{2} &
\end{array}
$$
$11 \mathrm{~B}) \quad c_{\mathrm{w}}=0.75$
if $\mathrm{b}=0-30^{\circ}$
$c_{w}=-0.01667 b+1.25$
if $\quad b=30-75^{\circ}$
$c_{w}=-0.02667 b+2.0$
if $\mathrm{b}=75-90^{\circ}$
$c_{w}=-0.4$
if $b=90-180^{\circ}$
$11 \mathrm{C}) \quad \mathrm{cp}_{\text {wind }}=1$
$\mathrm{cp}_{\mathrm{leo}}=-0.3$

TABLE 12: Equations used for temperature gradient

12A) $Q=\int_{H_{1}}^{H_{2}} D \Delta p|\Delta p|^{\frac{1}{n}-1}$

12 B) $\frac{d p}{d z}=-\frac{g p_{o} 273}{w_{z}+T_{z o}}$

12 C) $\quad \mathrm{p}=\frac{\mathrm{p}_{\mathrm{ref}}}{\mathrm{R}\left(\mathrm{T}_{\mathrm{ref}}-\lambda_{\mathrm{t}} \mathrm{H}\right)}$

and: $\quad \mathrm{dp}=-\rho \mathrm{g} \mathrm{dH}$

12 D) $T_{z}=a z+T_{z 0}$

and: $\quad p_{z}=g \int_{0}^{h} p(z) d z$ 
TABLE 13: Algorithm to solve the nonlinear set of equations

13A) $P_{j+1}=P_{j} \pm\left(\frac{Q_{j}}{C_{j} A_{j}}\right)^{n j}$

13 B) $\quad P_{j+1}=P_{j}-\frac{\Sigma Q}{\Sigma Q^{\prime}}$

$13 \mathrm{C}) \quad \mathrm{CR}=\frac{\sum \mathrm{D} \Delta \mathrm{P}^{1 / \mathrm{a}}}{\left.\Sigma \mid \frac{\mathrm{D}}{\mathrm{n}} \Delta \mathrm{P}^{((1 / \mathrm{n})-1)}\right\}}$

13 D) $\Sigma F=0$

13 E) $\quad P_{j+1}=P_{j}-$ Correction

$13 \mathrm{~F}) \quad$ Minimize over $P\left(\sum_{i=1}^{N} f^{2}(P)\right)$

with: $\quad f(P)=\sum_{j=1 ; i \neq j}^{N} L_{i j}$

$13 \mathrm{G}) \quad[\mathrm{A}][\Delta \mathrm{p}]=[\mathrm{q}]$

13 H) $\quad \mathrm{P}_{\mathrm{n}+1}=\mathrm{P}_{\mathrm{n}}-\lambda \frac{\mathrm{f}(\mathrm{p})}{\mathrm{J}(\mathrm{p})}$

13 I) $\quad \sum_{i} Q_{i}=0$ 
TABLE 14: Nomenclature

\begin{tabular}{|c|c|c|c|c|c|}
\hline Symbol & Description & Unit & Symbol & Description & Unit \\
\hline $\begin{array}{l}\mathrm{B} \\
\mathrm{C} \\
\mathrm{D} \\
\mathrm{E} \\
\mathrm{F} \\
\mathrm{H} \\
\mathrm{K} \\
\mathrm{L} \\
\mathrm{P} \\
\mathrm{Q} \\
\mathrm{R} \\
\mathrm{S} \\
\mathrm{T} \\
\mathrm{Zn}\end{array}$ & $\begin{array}{l}\text { area } \\
\text { constant } \\
\text { crack flow coefficient } \\
\text { flow coefficient } \\
\text { effective leakage area } \\
\text { mass flow rate } \\
\text { height } \\
\text { constant } \\
\text { crack length } \\
\text { pressure } \\
\text { volume flow rate } \\
\text { gas constant } \\
\text { resistance } \\
\text { absolute temperature } \\
\text { position of a neutral plane }\end{array}$ & $\begin{array}{c}\mathrm{m}^{2} \\
-- \\
\mathrm{m}^{3} / \mathrm{m} \mathrm{hPa}^{\mathrm{n}} \\
\mathrm{m}^{3} / \mathrm{h} \mathrm{Pa} \\
\mathrm{kg} / \mathrm{h} \\
\mathrm{kg} / \mathrm{h} \\
\mathrm{m} \\
-- \\
\mathrm{m} \\
\mathrm{Pa} \\
\mathrm{m}^{3} / \mathrm{h} \\
\mathrm{J} / \mathrm{kg} \mathrm{K} \\
\mathrm{h} \mathrm{Pa} / \mathrm{m}^{3} \\
\mathrm{~K} \\
\mathrm{~m}\end{array}$ & $\begin{array}{c}a, b, c \ldots \\
b_{\mathfrak{l}} \\
c p \\
d \\
f \\
f(\ldots) \\
g \\
\text { l } \\
n\end{array}$ & $\begin{array}{l}\text { coefficient } \\
\text { turbulent pressure gradient } \\
\text { surface pressure coefficient } \\
\text { diameter } \\
\text { friction factor } \\
\text { function of } \\
\text { gravity } \\
\text { duct length } \\
\text { flow exponent } \\
\text { relative temperature } \\
\text { velocity } \\
\text { wind speed } \\
\text { depth of crack }\end{array}$ & $\begin{array}{c}-- \\
\mathrm{Pa} / \mathrm{m} \\
-- \\
\mathrm{m} \\
-- \\
- \\
\mathrm{m} / \mathrm{s}^{2} \\
\mathrm{~m} \\
-- \\
{ }^{\circ} \mathrm{C} \\
\mathrm{m} / \mathrm{s} \\
\mathrm{m} / \mathrm{s} \\
\mathrm{m}\end{array}$ \\
\hline \multicolumn{3}{|c|}{ Greek letters } & \multicolumn{3}{|c|}{ Subscripts } \\
\hline $\begin{array}{l}\Delta \\
\sum \\
\Theta \\
\lambda \\
\lambda_{t} \\
\nu \\
\rho \\
\varsigma \\
\mu\end{array}$ & $\begin{array}{l}\text { difference } \\
\text { Summation } \\
\text { absolute temperature } \\
\text { friction factor } \\
\text { temperature gradient } \\
\text { viscosity } \\
\text { air density } \\
\text { fitting loss factor } \\
\text { viscosity of air }\end{array}$ & $\begin{array}{c}-- \\
-- \\
\mathrm{K} \\
-- \\
\mathrm{K} / \mathrm{m} \\
\mathrm{m}^{2} / \mathrm{s} \\
\mathrm{kg} / \mathrm{m}^{3} \\
-- \\
\mathrm{m}^{2} / \mathrm{s}\end{array}$ & $\begin{array}{c}j \\
j+1 \\
\text { NET } \\
o \\
r e f \\
s \\
t \\
0\end{array}$ & $\begin{array}{l}\text { discharge } \\
\text { locations in the duct system } \\
\text { inside } \\
\text { iteration step } \\
\text { iteration step + } 1 \\
\text { inside } \\
\text { outside } \\
\text { reference } \\
\text { stack } \\
\text { total } \\
\text { reference }\end{array}$ & $\begin{array}{l}-- \\
-- \\
-- \\
-- \\
-- \\
-- \\
-- \\
--\end{array}$ \\
\hline
\end{tabular}



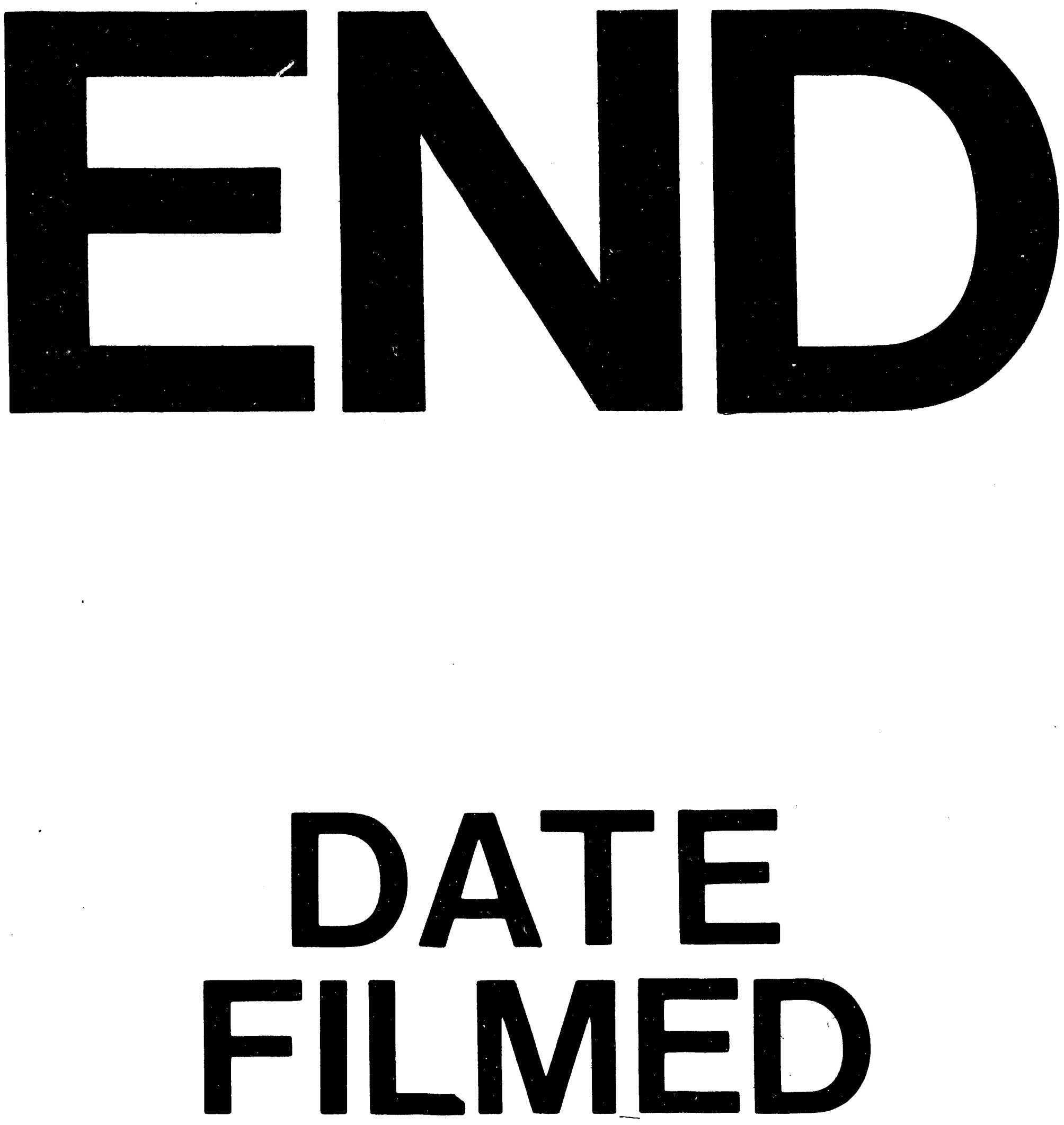

I

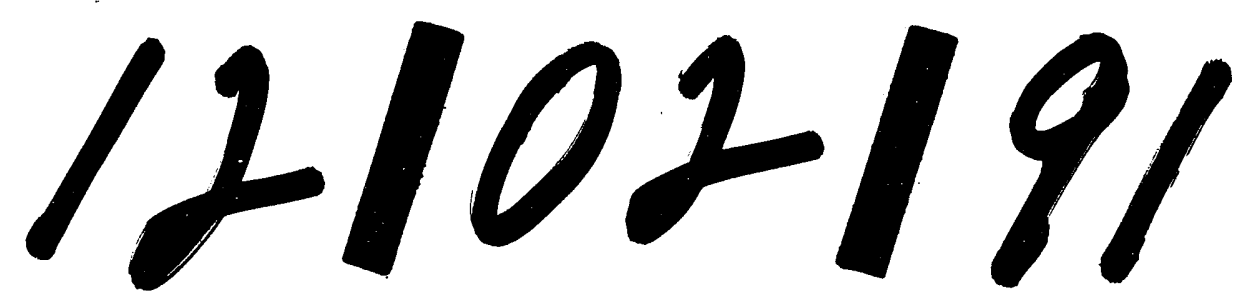


